\title{
Analysis, improvement and limits of the multiscale Latin method
}

\author{
Paul Oumaziz ${ }^{1,2}$, Pierre Gosselet ${ }^{3,4}$, Karin Saavedra $^{5}$, Nicolas Tardieu $^{6}$ \\ ${ }^{1}$ Núcleo Científico Multidisciplinario, Facultad de Ingeniería, Universidad de Talca, Campus Curicó, \\ Chile \\ ${ }^{2}$ Institut Clément Ader (ICA), Université de Toulouse, CNRS/INSA/ISAE/Mines Albi/UPS - 3 rue \\ Caroline Aigle, 31400 Toulouse, France \\ ${ }^{3}$ Univ. Lille, CNRS, Centrale Lille, UMR 9013 - LaMcube - Laboratoire de Mécanique, Multiphysique, \\ Multiéchelle, F-59000 Lille, France \\ ${ }^{4}$ LMT, ENS Paris-Saclay/UMR 8535 CNRS/Université Paris-Saclay - 4, avenue des Sciences 91190 \\ Gif-sur-Yvette, France \\ ${ }^{5}$ Dpto. Tecnologías Industriales, Facultad de Ingeniería, Universidad de Talca, Campus Curicó, Chile \\ ${ }^{6}$ IMSIA, UMR 9219 EDF-CNRS-CEA-ENSTA, Saclay, France
}

\begin{abstract}
This work studies the convergence properties of the mixed non-overlapping domain decomposition method (DDM) commonly named "Latin method". As all DDM, the Latin method is sensitive to nearinterface heterogeneity and irregularity. Using a simple yet fresh point of view, we analyze the role of the Robin parameters as well as of the second level (coarse space) correction - which are a characteristic of the method. In particular, we show how to build a spectrum-motivated coarse space aiming at ensuring fast convergence. 2D and 3D linear elasticity problems involving highly heterogeneous materials confirm the robustness of the spectral coarse space and provide evidence of the scalability of the multiscale Latin method.
\end{abstract}

Keywords Latin method, domain decomposition method, spectral coarse problem, GenEO, heterogeneous problem

\section{Introduction}

Domain Decomposition Methods [1] (DDM) offer a powerful framework to design efficient parallel iterative solvers for computational mechanics problems. Recently, much progress has been made in understanding the methods and how to make them robust, in particular with respect to misplaced heterogeneity and poorly shaped subdomains.

The first possibility consists in using a preprocessing step where local generalized eigenvalue problems detect "bad" spectral contributions. These harmful components are then removed from the resolution process by adding constraints to the system. In FETI-DP (Dual-Primal Finite-Element Tearing and Interconnection) or BDDC (Balancing Domain Decomposition by Constrains) methods, the constraints can be applied by selecting the coarse degrees of freedom [2, 3, 4, 5, 6, 7, 8]. In FETI and BDD methods, Krylov-augmented solvers are used to remove the bad modes which have been detected by a technique inspired from Schwarz methods (see [9, 10, 11, 12, 13] and references therein) and known as "Generalized Eigenvalue problems in the Overlap" (GenEO) [14, 15, 16, 17]. GenEO coarse spaces were also extended to the Symmetrized Optimized Restricted Additive Schwarz (SORAS) algorithm [18, 19]. 
The second alternative is to rely on a multipreconditioned solver [20]. This was proposed for the FETI method in 21] where as many search directions as subdomains are generated by the conjugate gradient at each iteration. Less demanding versions were presented in [22] and illustrated in [23]. Note that a link between multipreconditioning and GenEO coarse spaces was exhibited in [24].

This paper is devoted to the Latin DDM [25]. This strategy makes use of mixed (Robin) interface conditions, it was specifically designed to solve nonlinear mechanical problems involving complex behaviors inside subdomains and on their interfaces by stationary iterations, and it has been successfully applied to viscoplasticity, frictional contact, cracking, cohesive interfaces [26, 27, 28, 29, 30, 31] and also naturally coupled with the PGD (Proper Generalized Decomposition) model reduction [32, 33]. In [34, a two-level version was proposed to grant a certain scalability face to moderate heterogeneity. Note that in [35, 36], the authors proposed an analysis of the method in the light of more classical domain decomposition methods, and showed how a simple non-invasive implementation using python and MPI to pilot an industrial finite element software (code-aster [37] in this case)

Here, we propose to analyze the multiscale Latin approach and try to apply the GenEO philosophy. We show that local generalized eigenvalue problems naturally arise when studying the convergence of the Latin DDM. For a chosen convergence ratio, it is then possible to tell "nefarious" eigenvectors from "innocuous" ones, opening the path to sounder higher-order coarse spaces than the ones commonly based on polynomial development of interface quantities. Unfortunately, we show that in current state, the multiscale Latin approach does not permit to precisely remove the unwanted components and parasitic effects cannot be prevented.

The article is organized as follows: Section 2 introduces notations and the substructured problem, Section 3 presents the monoscale Latin method which is then analyzed in Section 4 through a generalized eigenvalue problem. Section 5 is dedicated to the multiscale (or two-level) approach, and we show how a hopefully efficient coarse space can be built from previously obtained eigenvector. Finally, Section 7 illustrates the method on three highly heterogeneous academic test-cases.

\section{The problem to be solved in substructured form}

A domain $\Omega \in \mathbb{R}^{d}$ ( $d=2$ or 3 ) under small perturbations and quasi-static isotherm evolution is considered, the material is supposed to be linear elastic and classical Lagrange conforming finite element is used to approximate the displacement field.

\section{$2.1 \quad$ Subdomains equations}

We take into account a non-overlapping decomposition of $N$ subdomains $\left(\Omega^{(s)}\right)_{S \in N}$. For the subdomain $\Omega^{(s)}$, let $\mathbf{K}^{(s)}$ denote the stiffness matrix, $\mathbf{u}^{(s)}$ the vector of nodal displacement and $\mathbf{g}^{(s)}$ the generalized forces associated to given loads, including the forces resulting from the elimination of Dirichlet boundary conditions. Matrix $\mathbf{K}^{(s)}$ is symmetric semi-definite positive, the null space is spanned by rigid body motions for floating subdomains.

Let $\Omega^{(s)}$ and $\Omega^{(q)}$ be two neighbors subdomains. We write $\partial^{(q)} \Omega^{(s)}$ the degrees of freedom of $\Omega^{(s)}$ facing $\Omega^{(q)}$. Some degrees of freedom can be shared by two boundaries, see Figure 1. Boundaries $\partial^{(q)} \Omega^{(s)}$ and $\partial^{(s)} \Omega^{(q)}$ interact through the interface $\Gamma^{\{s, q\}}=\partial \Omega^{(s)} \cap \partial \Omega^{(q)}$. The interface $\Gamma^{\{s, q\}}$ is granted its own finite element discretization, while displacements and reactions associated with each parent subdomains are defined as $\left(\mathbf{w}^{(s, q)}, \mathbf{w}^{(q, s)}\right)$ and $\left(\mathbf{f}^{(s, q)}, \mathbf{f}^{(q, s)}\right)$, respectively. These fields are connected to the parent subdomains by trace relations materialized by the operators $\mathbf{N}^{(s, q)}$ and $\mathbf{N}^{(q, s)}$. For now, we consider conforming discretization between the subdomains $\left(\Omega^{(s)}, \Omega^{(q)}\right)$ and the interface $\Gamma^{\{s, q\}}$, so that the trace operators are simple boolean matrices. Let $\mathcal{G}^{(s)}$ be the set of the neighbors of the subdomain $\Omega^{(s)} ; \mathcal{E}$ the set of all subdomains and $\mathcal{G}$ the set of all interfaces. 


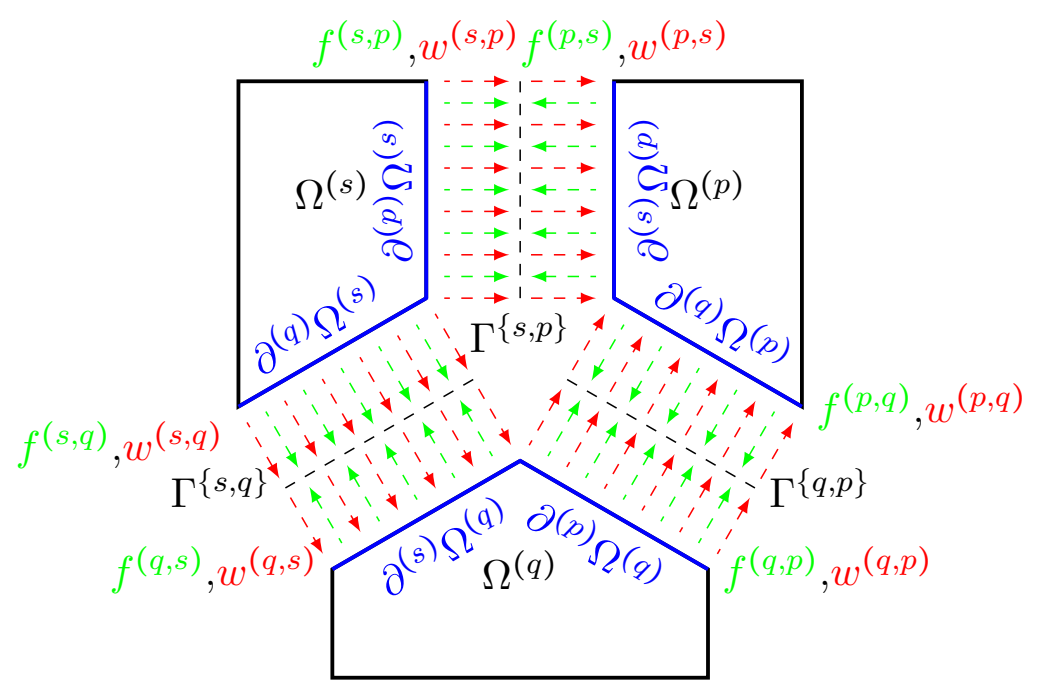

Figure 1: Force and displacement interface fields.

To easily handle the many interfaces of one subdomain $\Omega^{(s)}$, we define concatenated operators:

$$
\mathbf{w}^{(s)}=\left(\begin{array}{c}
\vdots \\
\mathbf{w}^{(s, q)} \\
\vdots
\end{array}\right), \quad \mathbf{f}^{(s)}=\left(\begin{array}{c}
\vdots \\
\mathbf{f}^{(s, q)} \\
\vdots
\end{array}\right), \quad \mathbf{N}^{(s)}=\left(\begin{array}{lll}
\ddots & & \\
& \mathbf{N}^{(s, q)} & \\
& & \ddots
\end{array}\right), \quad q \in \mathcal{G}^{(s)}
$$

Thus, the mechanical problems to be solved on the subdomains can be written as follows:

$$
\text { On subdomains: } \forall \Omega^{(s)} \in \mathcal{E}, \quad\left\{\begin{array}{l}
\mathbf{K}^{(s)} \mathbf{u}^{(s)}=\mathbf{g}^{(s)}+\mathbf{N}^{(s)^{T}} \mathbf{f}^{(s)} \\
\mathbf{w}^{(s)}=\mathbf{N}^{(s)} \mathbf{u}^{(s)}
\end{array}\right.
$$

Remark 1 (Condensation). Since the interface vectors are only connected to the boundary of the subdomains, we tag with index $b$ the boundary degrees of freedom of the subdomain and the trace relationship can be written as:

$$
\forall \Omega^{(s)} \in \mathcal{E}, \quad \mathbf{w}^{(s)}=\mathbf{N}_{b}^{(s)} \mathbf{u}_{b}^{(s)}
$$

Also, internal nodes (index $i$ ) can be eliminated from the balance of the domain leading to the condensed form of the equilibrium:

$$
\forall \Omega^{(s)} \in \mathcal{E}, \quad \mathbf{S}^{(s)} \mathbf{u}_{b}^{(s)}=\mathbf{b}^{(s)}+\mathbf{N}_{b}^{(s)^{T}} \mathbf{f}^{(s)}
$$

where $\mathbf{S}^{(s)}=\mathbf{K}_{b b}^{(s)}-\mathbf{K}_{b i}^{(s)} \mathbf{K}_{i i}^{(s)^{-1}} \mathbf{K}_{i b}^{(s)}$ is the Schur complement and $\mathbf{b}^{(s)}=\mathbf{g}_{b}^{(s)}-\mathbf{K}_{b i}^{(s)} \mathbf{K}_{i i}^{(s)^{-1}} \mathbf{g}_{i}$ is the condensed right-hand side.

Proposition 1 (Properties of $\mathbf{N}_{b}^{(s)}$ ). $\mathbf{N}_{b}^{(s)}$ is a $n_{\Gamma}^{(s)} \times n_{\partial}^{(s)}$ matrix, where $n_{\partial}^{(s)}$ is the number of boundary degrees of freedom of $\Omega^{(s)}$ and $n_{\Gamma}^{(s)}$ is the number of interface degrees of freedom of $\left(\Gamma^{\{s, q\}}\right)_{q \in \mathcal{G}^{(s)}}$. In the presence of multiple points (node facing more than one neighbor), $n_{\Gamma}^{(s)}>n_{\partial}^{(s)}$ and the matrix is not full-ranked, see Figure 1 .

\subsection{Interfaces equations}

The mechanical behavior of the interface $\Gamma^{\{s, q\}}$ is given by a relationship which links the boundary vectors $\left(\mathbf{w}^{(s, q)}, \mathbf{w}^{(q, s)}, \mathbf{f}^{(s, q)}, \mathbf{f}^{(q, s)}\right)$. Many mechanical behaviors, such as perfect interfaces, contact, friction or loss of cohesion can be represented by constraining the force to belong to a domain of admissibility - 
materialized by a function $\mathbf{c}^{-}$- which depends on the displacement gap and satisfies the action-reaction principle:

$$
\text { On general interfaces: } \forall \Gamma^{\{s, q\}} \in \mathcal{G},\left\{\begin{array}{l}
\mathbf{f}^{(s, q)} \in \mathbf{c}^{(s, q)}\left(\mathbf{w}^{(s, q)}-\mathbf{w}^{(q, s)}\right) \\
\mathbf{f}^{(s, q)}+\mathbf{f}^{(q, s)}=0
\end{array}\right.
$$

Note that internal variables could be incorporated into relation $\mathbf{c}$ to transcribe history-dependent behaviors like friction or debonding. However, in this work we only take into account perfect interfaces by stating the continuity of the displacement, then Equation (5) becomes:

$$
\text { On perfect interfaces: } \forall \Gamma^{\{s, q\}} \in \mathcal{G},\left\{\begin{array}{l}
\mathbf{w}^{(s, q)}=\mathbf{w}^{(q, s)} \\
\mathbf{f}^{(s, q)}+\mathbf{f}^{(q, s)}=0
\end{array}\right.
$$

\subsection{Assembly operators and interface behaviors}

Block notations are also here employed. For instance, vectors are concatenated by rows and matrices on the diagonal as follows:

$$
\mathbf{u}=\left(\begin{array}{c}
\vdots \\
\mathbf{u}^{(s)} \\
\vdots
\end{array}\right), \quad \mathbf{K}=\left(\begin{array}{lll}
\ddots & & 0 \\
& \mathbf{K}^{(s)} & \\
0 & & \ddots
\end{array}\right), \quad s \in \mathcal{E}
$$

This leads to the following expression being equivalent to Equation (2):

$$
\text { On subdomains: }\left\{\begin{array}{l}
\mathbf{K u}=\mathbf{g}+\mathbf{N}^{T} \mathbf{f} \\
\mathbf{w}=\mathbf{N u}
\end{array}\right.
$$

We introduce assembly operators for communicating neighboring subdomains. Operator $\mathbf{A}$ makes the sum of interface vectors whereas operator $\mathbf{B}$ makes the difference (an arbitrary orientation is chosen on the interface):

$$
\begin{aligned}
(\mathbf{A f})_{\mid \Gamma^{\{s, q\}}} & =\mathbf{f}^{(s, q)}+\mathbf{f}^{(q, s)} \\
(\mathbf{B w})_{\mid \Gamma^{\{s, q\}}} & =\mathbf{w}^{(s, q)}-\mathbf{w}^{(q, s)}
\end{aligned}
$$

Proposition 2 (Properties of interface operators). Operators $\mathbf{A}$ and $\mathbf{B}$ are $n_{\Gamma} \times 2 n_{\Gamma}$ matrices, where $n_{\Gamma}=\sum_{s<q} n_{\Gamma\{s, q\}}$ is the number of all interface degrees of freedom. They are full-ranked operators and also are orthogonal in the sense that:

$$
\operatorname{ker}(\mathbf{A})=\operatorname{range}\left(\mathbf{B}^{T}\right)
$$

This implies that $\mathbf{A B}^{T}=0$. More precisely, any interface vector $\mathbf{x}$ can be written uniquely under the form $\mathbf{x}=\mathbf{A}^{T} \mathbf{x}_{\mathbf{A}}+\mathbf{B}^{T} \mathbf{x}_{\mathbf{B}}$. In fact, this property can be generalized using non-euclidean orthogonality. Let $\mathbf{Q}$ be any symmetric positive definite matrix of size $2 n_{\Gamma}$, then we have:

$$
\mathbb{R}^{2 n_{\Gamma}}=\left(\mathbf{Q} \text { Range }\left(\mathbf{A}^{T}\right)\right) \oplus \operatorname{Range}\left(\mathbf{B}^{T}\right)
$$

Finally, assembly operators enable to easily write the perfect interface behavior as follows:

$$
\text { On perfect interfaces: }\left\{\begin{array}{l}
\mathbf{A f}=0 \\
\mathbf{B w}=0
\end{array}\right.
$$

Note that the general case takes the form $\mathbf{f}=\mathbf{B}^{T} \mathbf{c}(\mathbf{B w})$. This notation makes it clear that the interface equilibrium is satisfied since $\mathbf{A f}=\mathbf{A B}^{T} \mathbf{c}(\mathbf{B w})=0$. For instance, the contact formulation with Coulomb friction is fully detailed in [35]. 


\subsection{Partial solutions and total solution}

The interface fields are gathered in two sets:

$$
\begin{gathered}
\mathcal{A}:(\mathbf{w}, \mathbf{f}) \text { solutions to } \exists \mathbf{u} /\left\{\begin{array}{l}
\mathbf{K u}=\mathbf{g}+\mathbf{N}^{T} \mathbf{f} \\
\mathbf{w}=\mathbf{N u}
\end{array} \Leftrightarrow \exists \mathbf{u}_{b} /\left\{\begin{array}{l}
\mathbf{S} \mathbf{u}_{b}=\mathbf{b}+\mathbf{N}_{b}^{T} \mathbf{f} \\
\mathbf{w}=\mathbf{N}_{b} \mathbf{u}_{b}
\end{array}\right.\right. \\
\mathcal{L}:(\widehat{\mathbf{w}}, \widehat{\mathbf{f}}) \text { solutions to }\left\{\begin{array}{l}
\mathbf{A} \widehat{\mathbf{f}}=0 \\
\mathbf{B} \widehat{\mathbf{w}}=0
\end{array}\right.
\end{gathered}
$$

The set $\mathcal{A}$ generally called admissibility space is the affine space made out of the solutions to the linear equations on the subdomains, i.e. the equilibrium of the subdomains submitted to the given load. The set $\mathcal{L}$ of solutions to the interface equations is called local space; it is a vector space in the case of perfect interfaces, it would be a cone for Coulomb friction or a manifold for more complex interface behaviors.

Remark 2. Note that this framework extends to many mechanical situations, including time-dependent problems, nonlinear behaviors in the volume or large transformations, as long as $\mathcal{A}$ is characterized by linear equations (potentially associated with differential operators) and $\mathcal{L}$ is defined by non-differential equations (potentially nonlinear).

Of course, if $(\mathbf{w}, \mathbf{f}) \in \mathcal{L} \cap \mathcal{A}$ then $(\mathbf{w}, \mathbf{f})$ solves the whole substructured formulation. Note that $\mathbf{w}$ is defined uniquely but $\mathbf{f}$ is not as stated is the next proposition.

Proposition 3 (Indeterminacy of $\mathbf{f}$ ). In the presence a multiple points, the subspace $\operatorname{ker}\left(\mathbf{N}_{b}^{T} \mathbf{B}^{T}\right)$ is not reduced to 0 . This means that there exist balanced interface force (of the form $\mathbf{f}=\mathbf{B}^{T} \beta$ ) which result in the same mechanical effort $\mathbf{N}_{b}^{T} \mathbf{f}$ on the subdomains. This phenomenon is very well-known in FETI methods, and in practice it does not bring any difficulty.

\section{The monoscale Latin method}

\subsection{Principle}

The Latin method is an alternating direction algorithm. Solution $(\mathbf{w}, \mathbf{f})$ is reached through partial solutions of the sets $\mathcal{A}$ and $\mathcal{L}$, which are consecutively calculated as illustrated in Figure 2 .

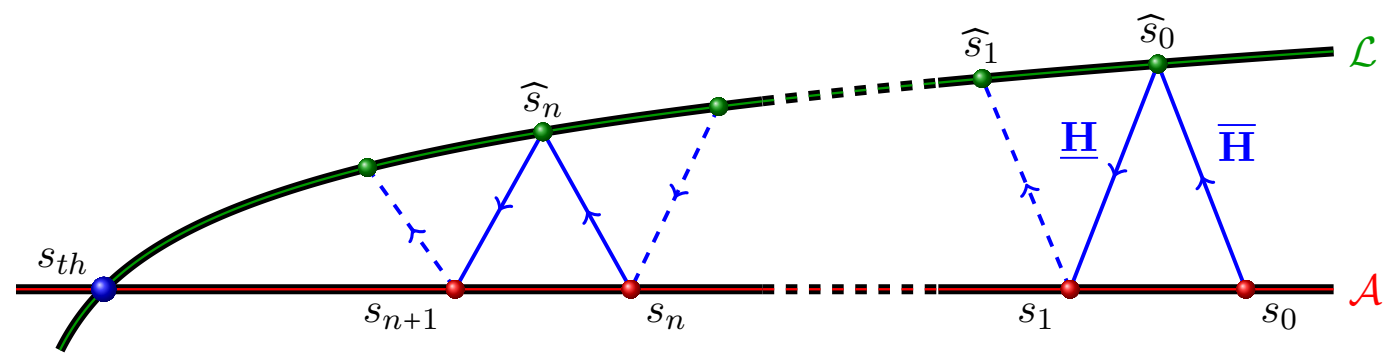

Figure 2: Illustration of the Latin framework.

Firstly, starting from a partial solution $s_{n}=\left(\mathbf{w}_{n}, \mathbf{f}_{n}\right) \in \mathcal{A}$, the so-called local stage consists in searching for a partial solution $\widehat{s}_{n}=\left(\widehat{\mathbf{w}}_{n}, \widehat{\mathbf{f}}_{n}\right) \in \mathcal{L}$. This is possible by enforcing a relation of the form:

$$
\widehat{\mathbf{f}}_{n}-\mathbf{f}_{n}-\overline{\mathbf{H}}\left(\widehat{\mathbf{w}}_{n}-\mathbf{w}_{n}\right)=0
$$

This equation is often called "local search direction" in the Latin literature. $\overline{\mathbf{H}}$ is a symmetric definite positive operator selected by the user, however, for computational efficiency - in particular in the case of complex interface behaviors - it should be chosen to be diagonal. 
Then, starting from a partial solution $\widehat{s}_{n}=\left(\widehat{\mathbf{w}}_{n}, \widehat{\mathbf{f}}_{n}\right) \in \mathcal{L}$, the so-called linear stage consists in searching for a partial solution $s_{n+1}=\left(\mathbf{w}_{n+1}, \mathbf{f}_{n+1}\right) \in \mathcal{A}$. This is possible by enforcing a relation of the form:

$$
\mathbf{f}_{n+1}-\widehat{\mathbf{f}}_{n}+\underline{\mathbf{H}}\left(\mathbf{w}_{n+1}-\widehat{\mathbf{w}}_{n}\right)=0
$$

This equation is often called "linear search direction", where $\underline{\mathbf{H}}$ is also a symmetric definite positive operator chosen by the user.

\subsection{The local stage}

The local stage finds a partial solution of the equations defined on the interfaces by enforcing the search direction. Then, the problem to be solved is:

$$
\text { Knowing } s_{n}=\left(\mathbf{w}_{n}, \mathbf{f}_{n}\right) \text {, find } \widehat{s}_{n}=\left(\widehat{\mathbf{w}}_{n}, \widehat{\mathbf{f}}_{n}\right) /\left\{\begin{array}{l}
\mathbf{A} \widehat{\mathbf{f}}_{n}=0 \\
\mathbf{B} \widehat{\mathbf{w}}_{n}=0 \\
\widehat{\mathbf{f}}_{n}-\mathbf{f}_{n}-\overline{\mathbf{H}}\left(\widehat{\mathbf{w}}_{n}-\mathbf{w}_{n}\right)=0
\end{array}\right.
$$

which gives the following solution:

$$
\left\{\begin{array}{l}
\widehat{\mathbf{w}}_{n}=\mathbf{A}^{T}\left(\mathbf{A} \overline{\mathbf{H}} \mathbf{A}^{T}\right)^{-1}\left[\mathbf{A} \overline{\mathbf{H}} \mathbf{w}_{n}-\mathbf{A} \mathbf{f}_{n}\right] \\
\widehat{\mathbf{f}}_{n}=\mathbf{B}^{T}\left(\mathbf{B} \overline{\mathbf{H}}^{-1} \mathbf{B}^{T}\right)^{-1}\left[\mathbf{B} \overline{\mathbf{H}}^{-1} \mathbf{f}_{n}-\mathbf{B} \mathbf{w}_{n}\right]
\end{array}\right.
$$

We recognize scaled assembly operators as classically encountered in FETI and BDD methods [38, 39]:

$$
\begin{aligned}
& \widehat{\mathbf{A}}=\left(\mathbf{A} \overline{\mathbf{H}} \mathbf{A}^{T}\right)^{-1} \mathbf{A} \overline{\mathbf{H}} \\
& \widehat{\mathbf{B}}=\left(\mathbf{B} \overline{\mathbf{H}}^{-1} \mathbf{B}^{T}\right)^{-1} \mathbf{B} \overline{\mathbf{H}}^{-1}
\end{aligned}
$$

They satisfy $\mathbf{A} \widehat{\mathbf{A}}^{T}=\mathbf{I}, \mathbf{B} \widehat{\mathbf{B}}^{T}=\mathbf{I}$ and $\mathbf{A}^{T} \widehat{\mathbf{A}}+\widehat{\mathbf{B}}^{T} \mathbf{B}=\mathbf{I}[40]$.

The set $\mathcal{L}$ gathers interface equations, and thus, compared to the complete system to be solved, it misses the subdomain mechanical behavior. $\overline{\mathbf{H}}$ should provide a computationally affordable ersatz of this information. To make this analysis precise, let us use the condensed form of $\mathcal{A}$ to express $\mathbf{w}$ and $\mathbf{f}$ in terms of $\mathbf{u}_{b}$.

$$
\begin{aligned}
\widehat{\mathbf{f}}_{n}-\mathbf{f}_{n}-\overline{\mathbf{H}}\left(\widehat{\mathbf{w}}_{n}-\mathbf{w}_{n}\right)=0 & \Rightarrow \mathbf{N}_{b}^{T} \widehat{\mathbf{f}}_{n}-\left(\mathbf{S u}_{b, n}-\mathbf{b}\right)-\mathbf{N}_{b}^{T} \overline{\mathbf{H}}\left(\widehat{\mathbf{w}}_{n}-\mathbf{w}_{n}\right)=0 \\
& \Rightarrow\left(\mathbf{N}_{b}^{T} \overline{\mathbf{H}} \mathbf{N}_{b}-\mathbf{S}\right) \mathbf{u}_{b, n}-\left(\mathbf{N}_{b}^{T} \overline{\mathbf{H}} \widehat{\mathbf{w}}_{n}-\mathbf{N}_{b}^{T} \widehat{\mathbf{f}}_{n}-\mathbf{b}\right)
\end{aligned}
$$

Proposition 4 (Optimal local search direction). If $\overline{\mathbf{H}}$ is such that $\mathbf{N}_{b}^{T} \overline{\mathbf{H}} \mathbf{N}_{b}=\mathbf{S}$, then the system formed by the above equation and the interface conditions (14) is equivalent to the substructured problem. In that case, the local stage directly gives the exact solution.

Let $\widetilde{\mathbf{N}}_{b}$ be a matrix such that $\mathbf{N}_{b}^{T} \widetilde{\mathbf{N}}_{b}=\mathbf{I}$, such as for instance $\widetilde{\mathbf{N}}_{b}=\mathbf{N}_{b}\left(\mathbf{N}_{b}^{T} \mathbf{N}_{b}\right)^{-1}$, and let $\widetilde{\mathbf{S}}$ be an approximation of $\mathbf{S}$ (like a weighted superlumped operator). We thus should choose $\overline{\mathbf{H}}=\operatorname{diag}\left(\widetilde{\mathbf{N}}_{b} \widetilde{\mathbf{S}}_{\mathbf{N}_{b}^{T}}^{T}\right)$. In the case of $\widetilde{\mathbf{N}}_{b}=\mathbf{N}_{b}\left(\mathbf{N}_{b}^{T} \mathbf{N}_{b}\right)^{-1}$, this accounts to weighting $\operatorname{diag}(\widetilde{\mathbf{S}})$ by the inverse of the multiplicity of the interface nodes (number of interfaces a boundary nodes textcolororangenode belongs to).

\subsection{The linear stage}

The linear stage finds a partial solution verifying the equilibria of subdomains while respecting the search directions. This leads to the following problem set independently on subdomains:

$$
\text { Knowing } \widehat{s}_{n}=\left(\widehat{\mathbf{w}}_{n}, \widehat{\mathbf{f}}_{n}\right) \text {, find } s_{n+1}=(\mathbf{w}, \mathbf{f}) /\left\{\begin{array}{l}
\mathbf{K} \mathbf{u}_{n+1}=\mathbf{g}+\mathbf{N}^{T} \mathbf{f}_{n+1} \\
\mathbf{w}_{n+1}=\mathbf{N u} \mathbf{u}_{n+1} \\
\mathbf{f}_{n+1}-\widehat{\mathbf{f}}_{n}+\underline{\mathbf{H}}\left(\mathbf{w}_{n+1}-\widehat{\mathbf{w}}_{n}\right)=0
\end{array}\right.
$$


leading as follows:

$$
\begin{aligned}
& \text { Solve for } \mathbf{u}_{n+1} \text { solution to: }\left(\mathbf{K}+\mathbf{N}^{T} \underline{\mathbf{H}}\right) \mathbf{u}_{n+1}=\mathbf{g}+\mathbf{N}^{T}\left(\widehat{\mathbf{f}}_{n}+\underline{\mathbf{H}} \widehat{\mathbf{w}}_{n}\right) \\
& \text { Then compute: }\left\{\begin{array}{c}
\mathbf{w}_{n+1}=\mathbf{N} \mathbf{u}_{n+1} \\
\mathbf{f}_{n+1}=\widehat{\mathbf{f}}_{n}+\underline{\mathbf{H}}\left(\widehat{\mathbf{w}}_{n}-\mathbf{w}_{n+1}\right)
\end{array}\right.
\end{aligned}
$$

\subsection{Relaxation}

To ensure convergence [25] a relaxation step is added at the end of a linear stage, thus:

$$
s_{n+1} \longleftarrow \omega s_{n+1}+(1-\omega) s_{n}
$$

The method is proved to converge in a very general framework (including nonlinear behavior associated with a convex potential) assuming that relaxation is used and that search directions are chosen to be equal $\overline{\mathbf{H}}=\underline{\mathbf{H}}$.

\subsection{Other choices of search directions}

Selecting the search directions is crucial for the convergence ratio of the algorithm. In Section 3.2 we have proposed approximations of the optimal one for the local stage, but we can also raise the possibility of search directions motivated by a FETI preconditioner [39]. In that case, the same direction is used for both stages, written $\mathbf{H}$ and defined as a scaled assembly of approximate subdomains stiffness. Additionally, the same value is employed for the two subdomains connected by an interface. Therefore, the common linear/local search direction can be written as:

$$
\mathbf{H}=\frac{1}{2}\left(\mathbf{A}^{T} \widetilde{\mathbf{H}} \mathbf{A}+\mathbf{B}^{T} \widetilde{\mathbf{H}} \mathbf{B}\right)
$$

where $\widetilde{\mathbf{H}}=\left(\mathbf{A D A}^{T}\right)^{-1} \mathbf{A D M D A} \mathbf{A}^{T}\left(\mathbf{A D A} \mathbf{A}^{T}\right)^{-1}$ is an operator defined on the interfaces, $\mathbf{D}$ a diagonal matrix and $\mathbf{M}$ a block-diagonal matrix. The operator $\frac{\mathbf{A}^{T} \ldots \mathbf{A}+\mathbf{B}^{T} \ldots \mathbf{B}}{2}$ brings back $\widetilde{\mathbf{H}}$ to the boundary of the subdomains.

In practice, inspired by FETI scaling and preconditioners, we propose to use $\mathbf{D}=\widetilde{\mathbf{N}}_{b} \operatorname{diag}\left(\mathbf{K}_{b b}\right)^{-1} \widetilde{\mathbf{N}}_{b}^{T}$, whereas two possible alternatives regarding $\mathbf{M}$ :

- $\mathbf{M}=\widetilde{\mathbf{N}}_{b} \operatorname{diag}(\mathbf{S})^{-1} \widetilde{\mathbf{N}}_{b}^{T}$, leading to a search direction named $\mathbf{H}_{S}$.

- $\mathbf{M}=\mathbf{D}^{+}$, leading to the search direction $\left.\mathbf{H}_{K}=\mathbf{A}^{T}(\mathbf{A D A})^{T}\right)^{-1} \mathbf{A}$.

\section{Analysis of the monoscale method}

In this section, the Latin method is recast into a fixed-point iteration and a convergence analysis is then derived. This allows to recover some classical properties of the algorithm and to introduce better decisions for a multiscale extension.

Because the equations of perfect interfaces are linear, we can rewrite both linear and local stages in a matrix form with the interface unknown $\left(\mathbf{w}_{n}, \mathbf{f}_{n}\right)$ and $\left(\widehat{\mathbf{w}}_{n}, \widehat{\mathbf{f}}_{n}\right)$ as the variables, where index $n$ stands for the iteration counter. In fact, the local stage 18 can be written as:

$$
\left(\begin{array}{c}
\widehat{\mathbf{w}}_{n} \\
\widehat{\mathbf{f}}_{n}
\end{array}\right)=\left(\begin{array}{cc}
\mathbf{A}^{T} \widehat{\mathbf{A}} & 0 \\
0 & \mathbf{B}^{T} \widehat{\mathbf{B}}
\end{array}\right)\left(\begin{array}{c}
\overline{\mathbf{H}}^{-1} \\
-\mathbf{I}
\end{array}\right)\left(\begin{array}{ll}
\overline{\mathbf{H}} & -\mathbf{I}
\end{array}\right)\left(\begin{array}{c}
\mathbf{w}_{n} \\
\mathbf{f}_{n}
\end{array}\right)
$$

while the linear stage 22 can be formulated like this:

$$
\left(\begin{array}{c}
\mathbf{w}_{n+1} \\
\mathbf{f}_{n+1}
\end{array}\right)=\left(\begin{array}{c}
\mathbf{w}_{0} \\
\mathbf{f}_{0}
\end{array}\right)+\left(\begin{array}{cc}
\mathbf{I} & 0 \\
-\underline{\mathbf{H}} & \mathbf{I}
\end{array}\right)\left(\begin{array}{c}
\mathbf{N}\left(\mathbf{K}+\mathbf{N}^{T} \underline{\mathbf{H}} \mathbf{N}\right)^{-1} \mathbf{N}^{T} \\
\mathbf{I}
\end{array}\right)\left(\begin{array}{ll}
\underline{\mathbf{H}} & \mathbf{I}
\end{array}\right)\left(\begin{array}{c}
\widehat{\mathbf{w}}_{n} \\
\widehat{\mathbf{f}}_{n}
\end{array}\right)
$$


where, we have named the following quantity:

$$
\left(\begin{array}{c}
\mathbf{w}_{0} \\
\mathbf{f}_{0}
\end{array}\right)=\left(\begin{array}{c}
\mathbf{I} \\
-\underline{\mathbf{H}}
\end{array}\right) \mathbf{N}\left(\mathbf{K}+\mathbf{N}^{T} \underline{\mathbf{H}} \mathbf{N}\right)^{-1} \mathbf{g}
$$

At last, if we introduce the mixed variable $\boldsymbol{\mu}_{n}=\overline{\mathbf{H}} \mathbf{w}_{n}-\mathbf{f}_{n}$, the iteration takes the form $\boldsymbol{\mu}_{n+1}=\boldsymbol{\mu}_{0}+\mathbf{M} \boldsymbol{\mu}_{n}$, where the iteration matrix $\mathbf{M}$ can be written as:

$$
\mathbf{M}=\left(\begin{array}{ll}
\overline{\mathbf{H}} & -\mathbf{I}
\end{array}\right)\left(\begin{array}{cc}
\mathbf{I} & 0 \\
-\underline{\mathbf{H}} & \mathbf{I}
\end{array}\right)\left(\begin{array}{c}
\mathbf{N}\left(\mathbf{K}+\mathbf{N}^{T} \underline{\mathbf{H}} \mathbf{N}\right)^{-1} \mathbf{N}^{T} \\
\mathbf{I}
\end{array}\right) \ldots
$$

$$
\ldots\left(\begin{array}{ll}
\underline{\mathbf{H}} & \mathbf{I}
\end{array}\right)\left(\begin{array}{cc}
\mathbf{A}^{T} \widehat{\mathbf{A}} & 0 \\
0 & \mathbf{B}^{T} \widehat{\mathbf{B}}
\end{array}\right)\left(\begin{array}{c}
\overline{\mathbf{H}}^{-1} \\
-\mathbf{I}
\end{array}\right)
$$

Using the expression of the scaling matrices 19 and their properties, it simplifies to:

$$
\begin{aligned}
\mathbf{M} & =\left((\overline{\mathbf{H}}+\underline{\mathbf{H}}) \mathbf{N}\left(\mathbf{K}+\mathbf{N}^{T} \underline{\mathbf{H}} \mathbf{N}\right)^{-1} \mathbf{N}^{T}-\mathbf{I}\right)\left(\underline{\mathbf{H}} \mathbf{A}^{T} \widehat{\mathbf{A}} \overline{\mathbf{H}}^{-1}-\mathbf{B}^{T} \widehat{\mathbf{B}}\right) \\
& =\underbrace{\left((\overline{\mathbf{H}}+\underline{\mathbf{H}}) \mathbf{N}\left(\mathbf{K}+\mathbf{N}^{T} \underline{\mathbf{H}} \mathbf{N}\right)^{-1} \mathbf{N}^{T}-\mathbf{I}\right)}_{\mathbf{M}_{1}} \underbrace{\left.(\underline{\mathbf{H}}+\overline{\mathbf{H}}) \mathbf{A}^{T}\left(\mathbf{A} \overline{\mathbf{H}} \mathbf{A}^{T}\right)^{-1} \mathbf{A}-\mathbf{I}\right)}_{\mathbf{M}_{2}}
\end{aligned}
$$

The iteration matrix can thus be written as the product of two matrices of which we suggest analyze the spectrum under the next hypotheses:

Hypothesis 1 (Same assembled search directions). $\mathbf{A} \overline{\mathbf{H}} \mathbf{A}^{T}=\mathbf{A} \underline{\mathbf{H}} \mathbf{A}^{T}$.

Hypothesis 2 (Identical search directions). $\overline{\mathbf{H}}=\underline{\mathbf{H}}$, in that case we use the notation $\mathbf{H}$ for both directions. Of course, Hypothesis 2 is stronger than Hypothesis 1 .

Proposition 5 (Spectrum of matrix $\mathbf{M}_{2}$ ). Under Hypothesis 1 , the spectrum of Matrix $\mathbf{M}_{2}$ is exactly $\{-1,1\}$ and thus the spectral radius is 1 .

Proof. Let be $\mathbf{x}^{T}=\left(\mathbf{x}_{A}^{T} \mathbf{A}+\mathbf{x}_{B}^{T} \mathbf{B}\right)$. We have:

$$
\begin{aligned}
\mathbf{x}_{A}^{T} \mathbf{A} \mathbf{M}_{2} & =\mathbf{x}_{A}^{T} \mathbf{A}(\underline{\mathbf{H}}+\overline{\mathbf{H}}) \mathbf{A}^{T}\left(\mathbf{A} \overline{\mathbf{H}} \mathbf{A}^{T}\right)^{-1}-\mathbf{x}_{A}^{T} \mathbf{A} \\
& =2 \mathbf{x}_{A}^{T} \mathbf{A}-\mathbf{x}_{A}^{T} \mathbf{A}=\mathbf{x}_{A}^{T} \mathbf{A} \Rightarrow \lambda=1 \\
\mathbf{x}_{B}^{T} \mathbf{B}(\underline{\mathbf{H}}+\overline{\mathbf{H}})^{-1} \mathbf{M}_{2} & =\mathbf{x}_{B}^{T} \mathbf{B} \mathbf{A}^{T}\left(\mathbf{A} \overline{\mathbf{H}} \mathbf{A}^{T}\right)^{-1} \mathbf{A}-\mathbf{x}_{B}^{T} \mathbf{B}(\underline{\mathbf{H}}+\overline{\mathbf{H}})^{-1} \\
& =-\mathbf{x}_{B}^{T} \mathbf{B}(\underline{\mathbf{H}}+\overline{\mathbf{H}})^{-1} \Rightarrow \lambda=-1
\end{aligned}
$$

We thus have found a family of left eigenvectors for matrix $\mathbf{M}_{2}$, thanks to Proposition 2 they span the whole space.

Proposition 6 (Spectrum of matrix $\mathbf{M}_{1}$ ). Under Hypothesis 2, the spectrum of Matrix $\mathbf{M}_{1}$ is in the interval ] - 1,1], the eigenvalue 1 is associated with rigid body motions of floating subdomains.

Proof. Let $\lambda$ be an eigenvalue of $\mathbf{M}_{1}$ and $\mathbf{x}$ an associated eigenvector, thus:

$$
\begin{aligned}
\mathbf{M}_{1} \mathbf{x}=\left((\mathbf{H}+\mathbf{H}) \mathbf{N}\left(\mathbf{K}+\mathbf{N}^{T} \underline{\mathbf{H}} \mathbf{N}\right)^{-1} \mathbf{N}^{T}-\mathbf{I}\right) \mathbf{x}=\lambda \mathbf{x} \\
\mathbf{H N}\left(\mathbf{K}+\mathbf{N}^{T} \mathbf{H N}\right)^{-1} \mathbf{N}^{T} \mathbf{x}=\frac{(\lambda+1)}{2} \mathbf{x} \\
\mathbf{N}^{T} \mathbf{H N}\left(\mathbf{K}+\mathbf{N}^{T} \mathbf{H N}\right)^{-1} \mathbf{N}^{T} \mathbf{x}=\frac{(\lambda+1)}{2} \mathbf{N}^{T} \mathbf{x}
\end{aligned}
$$


If we let $\mathbf{y}=\left(\mathbf{K}+\mathbf{N}^{T} \mathbf{H N}\right)^{-1} \mathbf{N}^{T} \mathbf{x}$, we have:

$$
\begin{aligned}
\mathbf{N}^{T} \mathbf{H N y} & =\frac{(\lambda+1)}{2}\left(\mathbf{K}+\mathbf{N}^{T} \mathbf{H N}\right) \mathbf{y} \\
\lambda & =2 \frac{\mathbf{y}^{T} \mathbf{N}^{T} \mathbf{H} \mathbf{N y}}{\mathbf{y}^{T} \mathbf{K y}+\mathbf{y}^{T} \mathbf{N}^{T} \mathbf{H N y}}-1
\end{aligned}
$$

The bounds are obtained by observing that $\mathbf{y}^{T} \mathbf{K y} \geqslant 0$ and $\mathbf{y}^{T} \mathbf{N}^{T} \mathbf{H N y}>0$. The value $\lambda=1$ is obtained for $\mathbf{K} \mathbf{y}=0$ which means that $\mathbf{y}$ is a (subdomain-wise) rigid body motion.

From this analysis we can conclude that the iteration is a contraction, relaxation is required if there exist floating subdomains.

\section{The multiscale Latin method}

In this section we briefly present the multiscale extension of the Latin method [34] which is closely connected to classical domain decomposition methods' coarse problems.

\subsection{Principle}

The multiscale extension consists in back-porting part of the interface equations in the subdomain stage. The action-reaction principle $\mathbf{A f}=0$ has the advantage to be a linear equation satisfied even by complex nonlinear interface behavior. We wish to ensure that the action-reaction principle is at least satisfied perpendicularly to a subspace of interface displacements. This subspace is often referred to as the subspace of "macro displacements" and it is given by a basis $\mathbb{W}^{M}$.

Remark 3 (Usual macrospace). In the Latin-method literature, the macro-space is often defined in each interface. $\mathbb{W}^{M}$ is constituted at least by interfaces' rigid body motions, often low-order deformation modes are added (simple strain) or, in some cases, higher-degree polynomial displacements are added [41.

In the multiscale framework, the linear stage (21) becomes:

Knowing $\widehat{s}_{n}=\left(\widehat{\mathbf{w}}_{n}, \widehat{\mathbf{f}}_{n}\right)$, find $s_{n+1}=\left(\mathbf{w}_{n+1}, \mathbf{f}_{n+1}\right)$ such that:

$$
\left\{\begin{array}{lr}
\mathbf{K} \mathbf{u}_{n+1}=\mathbf{g}+\mathbf{N}^{T} \mathbf{f}_{n+1} & \text { balance of subdomains } \\
\mathbf{w}_{n+1}=\mathbf{N} \mathbf{u}_{n+1} & \text { trace relationship } \\
\mathbb{W}^{M^{T}} \mathbf{A} \mathbf{f}_{n+1}=0 & \text { macro constraint } \\
\mathbf{f}_{n+1}-\widehat{\mathbf{f}}_{n}+\underline{\mathbf{H}}\left(\mathbf{w}_{n+1}-\widehat{\mathbf{w}}_{n}-\mathbf{A}^{T} \mathbb{W}^{M} \boldsymbol{\alpha}_{n+1}\right)=0 & \text { search direction }
\end{array}\right.
$$

where the search direction was modified in order to comply with the macro constraint. It was shown in [36] that the multiscale extension simply amounts to modifying the linear search direction, as follows:

Knowing $\widehat{s}_{n}=\left(\widehat{\mathbf{w}}_{n}, \widehat{\mathbf{f}}_{n}\right)$, find $s_{n+1}=\left(\mathbf{w}_{n+1}, \mathbf{f}_{n+1}\right)$ such that:

$$
\left\{\begin{array}{l}
\mathbf{K} \mathbf{u}_{n+1}=\mathbf{g}+\mathbf{N}^{T} \mathbf{f}_{n+1} \\
\mathbf{w}_{n+1}=\mathbf{N u} \mathbf{u}_{n+1} \\
\mathbf{f}_{n+1}-\widehat{\mathbf{f}}_{n}+\underline{\mathbf{H}}(\mathbf{I}-\mathbf{P})\left(\mathbf{w}_{n+1}-\widehat{\mathbf{w}}_{n}\right)=0 \\
\text { with } \mathbf{P}=\mathbf{A}^{T} \mathbb{W}^{M}\left(\mathbb{W}^{M^{T}} \mathbf{A} \underline{\mathbf{H}} \mathbf{A}^{T} \mathbb{W}^{M}\right)^{-1} \mathbb{W}^{M^{T}} \mathbf{A} \underline{\mathbf{H}}
\end{array}\right.
$$

Proposition 7 (Properties of $\mathbf{P}$ ). $\mathbf{P}$ is a projector satisfying the following relationships:

$$
\begin{array}{rlrl}
\underline{\mathbf{H}} \mathbf{P} & =\mathbf{P}^{T} \underline{\mathbf{H}}, & \mathbf{P A}^{T} \mathbb{W}^{M} & =\mathbf{A}^{T} \mathbb{W}^{M}, \\
\mathbf{B P} & =0, & (\mathbf{I}-\mathbf{P})^{T} \underline{\mathbf{H}} \mathbf{P}=0
\end{array}
$$


Projector $\mathbf{P}$ can be used to extract the so-called macro part of an interface displacement $\mathbf{P} \mathbf{w}$ and of an interface effort $\mathbf{P}^{T} \mathbf{f}$.

\subsection{Linear stage}

When using the projection $(\mathbf{I}-\mathbf{P})$ into the search direction, is similar to softening the stiffness $\underline{\mathbf{H}}$. It also introduces a low-rank coupling between neighbors subdomains, which is exploited by using the ShermanMorrison formula. In fact, if we set:

$$
\left(\begin{array}{c}
\mathbf{w}_{0} \\
\mathbf{f}_{0}
\end{array}\right)=\left(\begin{array}{c}
\mathbf{I} \\
-\underline{\mathbf{H}}(\mathbf{I}-\mathbf{P})
\end{array}\right) \mathbf{N}\left(\mathbf{K}+\mathbf{N}^{T} \underline{\mathbf{H}}(\mathbf{I}-\mathbf{P}) \mathbf{N}\right)^{-1} \mathbf{g}
$$

The linearstage $(26)$ can be written as:

$$
\left(\begin{array}{c}
\mathbf{w}_{n+1} \\
\mathbf{f}_{n+1}
\end{array}\right)=\left(\begin{array}{c}
\mathbf{w}_{0} \\
\mathbf{f}_{0}
\end{array}\right)+\left(\begin{array}{cc}
\mathbf{I} & 0 \\
-\underline{\mathbf{H}}(\mathbf{I}-\mathbf{P}) & \mathbf{I}
\end{array}\right) \cdots
$$

$$
\ldots\left(\begin{array}{c}
\mathbf{N}\left(\mathbf{K}+\mathbf{N}^{T} \underline{\mathbf{H}}(\mathbf{I}-\mathbf{P}) \mathbf{N}\right)^{-1} \mathbf{N}^{T} \\
\mathbf{I}
\end{array}\right)(\underline{\mathbf{H}}(\mathbf{I}-\mathbf{P}) \quad \mathbf{I})\left(\begin{array}{l}
\widehat{\mathbf{w}}_{n} \\
\widehat{\mathbf{f}}_{n}
\end{array}\right)
$$

We observe the fundamental property $\mathbf{P}^{T} \mathbf{f}_{n}=0$ meaning that the effort is purely microscopic. Besides, only the micro part of the displacement from the local stage $(\mathbf{I}-\mathbf{P}) \widehat{\mathbf{w}}_{n}$ is used as an input.

\subsection{Local stage under Hypothesis 2}

The new iteration matrix can be obtained by simply replacing $\underline{\mathbf{H}}$ by $\underline{\mathbf{H}}(\mathbf{I}-\mathbf{P})$ in 28$)$. However it is hard to derive further analysis in the general case, thus we restrict our analysis to Hypothesis 2 .

If $\underline{\mathbf{H}}=\overline{\mathbf{H}}=\mathbf{H}$, important simplifications also occur in the local stage. Indeed, $\mathbf{A} \widehat{\mathbf{f}}_{n}=0$ implies that there exists some $\beta$ such that $\widehat{\mathbf{f}}_{n}=\mathbf{B}^{T} \beta$ thus $\mathbf{P}^{T} \widehat{\mathbf{f}}_{n}=\mathbf{P}^{T} \mathbf{B}^{T} \beta=0$; together with the properties of $\mathbf{P}$ and $\mathbf{f}_{n}$, the search direction $(15)$ can be written as:

$$
\begin{aligned}
0=\widehat{\mathbf{f}}_{n}-\mathbf{f}_{n}-\mathbf{H}\left(\widehat{\mathbf{w}}_{n}-\mathbf{w}_{n}\right) & =\left(\mathbf{I}-\mathbf{P}^{T}\right)\left(\widehat{\mathbf{f}}_{n}-\mathbf{f}_{n}\right)-\mathbf{H}(\mathbf{I}-\mathbf{P}+\mathbf{P})\left(\widehat{\mathbf{w}}_{n}-\mathbf{w}_{n}\right) \\
& =\left(\mathbf{I}-\mathbf{P}^{T}\right)\left(\left(\widehat{\mathbf{f}}_{n}-\mathbf{f}_{n}\right)-\mathbf{H}\left(\widehat{\mathbf{w}}_{n}-\mathbf{w}_{n}\right)\right)-\mathbf{H P}\left(\widehat{\mathbf{w}}_{n}-\mathbf{w}_{n}\right) \\
\Longrightarrow & \Longrightarrow\left\{\begin{array}{l}
0=\left(\mathbf{I}-\mathbf{P}^{T}\right)\left(\left(\widehat{\mathbf{f}}_{n}-\mathbf{f}_{n}\right)-\mathbf{H}\left(\widehat{\mathbf{w}}_{n}-\mathbf{w}_{n}\right)\right) \\
0=\mathbf{P}\left(\widehat{\mathbf{w}}_{n}-\mathbf{w}_{n}\right)
\end{array}\right.
\end{aligned}
$$

This means that the macro part of displacements is preserved during the local stage, whereas the

micro part of efforts and displacements are coupled by $\mathbf{H}(\mathbf{I}-\mathbf{P})$. Therefore, the local stage (25) can be transformed into the matrix form:

$$
\left(\begin{array}{c}
\mathbf{P} \widehat{\mathbf{w}}_{n} \\
(\mathbf{I}-\mathbf{P}) \widehat{\mathbf{w}}_{n} \\
\widehat{\mathbf{f}}_{n}
\end{array}\right)=\left(\begin{array}{ccc}
\mathbf{I} & 0 & 0 \\
0 & (\mathbf{I}-\mathbf{P}) \mathbf{A}^{T} \widehat{\mathbf{A}} & -(\mathbf{I}-\mathbf{P}) \mathbf{A}^{T} \widehat{\mathbf{A}} \mathbf{H}^{-1} \\
0 & -\mathbf{B}^{T} \widehat{\mathbf{B}} \mathbf{H} & \mathbf{B}^{T} \widehat{\mathbf{B}}
\end{array}\right)\left(\begin{array}{c}
\mathbf{P w}_{n} \\
(\mathbf{I}-\mathbf{P}) \mathbf{w}_{n} \\
\mathbf{f}_{n}
\end{array}\right)
$$

\subsection{Multiscale iteration matrix under Hypothesis 2}

If we gather previous results, the iteration is driven by the micro mixed variable $\boldsymbol{\mu}_{n}=\mathbf{H}(\mathbf{I}-\mathbf{P}) \mathbf{w}_{n}+\mathbf{f}_{n}$ and the iteration matrix $\mathbf{M}$ can turned into:

$$
\begin{aligned}
& \mathbf{M}=\left(\begin{array}{ll}
\mathbf{H} & -\mathbf{I}
\end{array}\right)\left(\begin{array}{cc}
(\mathbf{I}-\mathbf{P}) & 0 \\
-\mathbf{H}(\mathbf{I}-\mathbf{P}) & \mathbf{I}
\end{array}\right)\left(\begin{array}{c}
\mathbf{N}\left(\mathbf{K}+\mathbf{N}^{T} \mathbf{H}(\mathbf{I}-\mathbf{P}) \mathbf{N}\right)^{-1} \mathbf{N}^{T} \\
\mathbf{I}
\end{array}\right) \ldots \\
& \ldots\left(\begin{array}{ll}
\mathbf{H} & \mathbf{I}
\end{array}\right)\left(\begin{array}{cc}
(\mathbf{I}-\mathbf{P}) \mathbf{A}^{T} \widehat{\mathbf{A}} & 0 \\
0 & \mathbf{B}^{T} \widehat{\mathbf{B}}
\end{array}\right)\left(\begin{array}{c}
\mathbf{H}^{-1} \\
-\mathbf{I}
\end{array}\right)
\end{aligned}
$$


and if we use $\mathbf{P}^{T} \mathbf{B}^{T}=0$ and $\mathbf{P}^{T} \mathbf{H}=\mathbf{H P}$ this simplifies to:

$$
\mathbf{M}=\left(\mathbf{I}-\mathbf{P}^{T}\right) \underbrace{\left(2 \mathbf{H N}\left(\mathbf{K}+\mathbf{N}^{T} \mathbf{H}(\mathbf{I}-\mathbf{P}) \mathbf{N}\right)^{-1} \mathbf{N}^{T}-\mathbf{I}\right)}_{\mathbf{M}_{3}} \ldots \ldots \ldots\left(\mathbf{I}-\mathbf{P}^{T}\right) \underbrace{\left(2 \mathbf{H A}^{T}\left(\mathbf{A H A}^{T}\right)^{-1} \mathbf{A}-\mathbf{I}\right)}_{\mathbf{M}_{2}}
$$

where matrix $\mathbf{M}_{2}$ is unchanged from one defined in the monoscale iteration matrix 28 .

\section{Analysis of the multiscale method under Hypothesis 2}

We consider the eigenproblems that drives the convergence of the monoscale method, let $\boldsymbol{\Lambda}$ be the diagonal matrix of eigenvalues. From the generalized eigenvalue problems with symmetric definite positive matrices (31), we can build the basis of eigenvectors $\mathbf{Y}$ which satisfies:

$$
\begin{aligned}
\left(\mathbf{N}^{T} \mathbf{H N}\right) \mathbf{Y} & =\left(\mathbf{K}+\mathbf{N}^{T} \mathbf{H N}\right) \mathbf{Y} \frac{(\boldsymbol{\Lambda}+\mathbf{I})}{2} \\
\mathbf{Y}^{T}\left(\mathbf{N}^{T} \mathbf{H N}\right) \mathbf{Y} & =\frac{(\boldsymbol{\Lambda}+\mathbf{I})}{2} \\
\mathbf{Y}^{T}\left(\mathbf{K}+\mathbf{N}^{T} \mathbf{H N}\right) \mathbf{Y} & =\mathbf{I}
\end{aligned}
$$

Note that we can recover the original eigenvectors of $30 p$ :

$$
\begin{aligned}
\mathbf{X} & =\mathbf{H N}\left(\mathbf{N}^{T} \mathbf{H N}\right)^{-1}\left(\mathbf{K}+\mathbf{N}^{T} \mathbf{H N}\right) \mathbf{Y} \\
& =\mathbf{H N Y} 2(\boldsymbol{\Lambda}+\mathbf{I})^{-1} \\
\mathbf{X} \frac{(\boldsymbol{\Lambda}+\mathbf{I})}{2} & =\mathbf{H N}\left(\mathbf{K}+\mathbf{N}^{T} \mathbf{H N}\right)^{-1} \mathbf{N}^{T} \mathbf{X}
\end{aligned}
$$

Considering the iteration matrix 39 , the convergence of the multiscale method is driven by the following eigenproblem:

$$
\left(\mathbf{I}-\mathbf{P}^{T}\right) \underbrace{\left(2 \mathbf{H N}\left(\mathbf{K}+\mathbf{N}^{T} \mathbf{H}(\mathbf{I}-\mathbf{P}) \mathbf{N}\right)^{-1} \mathbf{N}^{T}-\mathbf{I}\right)}_{\mathbf{M}_{3}}\left(\mathbf{I}-\mathbf{P}^{T}\right) \mathbf{x}=\gamma \mathbf{x}
$$

Proposition 8 (Spectral stability under macro constraint). Let $\mathbf{x}$ be an eigenvector of $\mathbf{M}_{1}$ as given in (30). Assume $\mathbf{x} \in \operatorname{Ker}\left(\mathbf{P}^{T}\right)=\operatorname{Range}\left(\mathbf{I}-\mathbf{P}^{T}\right)$, then $\mathbf{x}$ is an eigenvector of $\mathbf{M}_{3}$ and the eigenvalue is unchanged.

Proof. We note $\widetilde{\mathbf{K}}=\left(\mathbf{K}+\mathbf{N}^{T} \mathbf{H N}\right)$, let $\lambda$ be the eigenvalue of 30 associated with $\mathbf{x}$. We have:

$$
\mathbf{H N} \widetilde{\mathbf{K}}^{-1} \mathbf{N}^{T} \mathbf{x}=\frac{(\boldsymbol{\lambda}+1)}{2} \mathbf{x}
$$

We note $\mathbf{Z}=\mathbf{N}^{T} \mathbf{H} \mathbf{A}^{T} \mathbb{W}^{M}$ and use the Sherman-Morrison formula to develop $\mathbf{M}_{3}$ :

$$
\begin{aligned}
\frac{\mathbf{M}_{3}+\mathbf{I}}{2} & =\mathbf{H N}\left(\widetilde{\mathbf{K}}-\mathbf{Z}\left(\mathbb{W}^{M^{T}} \mathbf{A} \mathbf{H} \mathbf{A}^{T} \mathbb{W}^{M}\right)^{-1} \mathbf{Z}^{T}\right)^{-1} \mathbf{N}^{T} \\
& =\mathbf{H N}\left(\widetilde{\mathbf{K}}^{-1}+\widetilde{\mathbf{K}}^{-1} \mathbf{Z}\left(\mathbb{W}^{M^{T}} \mathbf{A}\left(\mathbf{H}-\mathbf{H} \mathbf{N} \widetilde{\mathbf{K}}^{-1} \mathbf{N}^{T} \mathbf{H}\right)^{-1} \mathbf{A}^{T} \mathbb{W}^{M}\right) \mathbf{Z}^{T} \widetilde{\mathbf{K}}^{-1}\right) \mathbf{N}^{T}
\end{aligned}
$$

Since $\mathbf{x}$ is an eigenvector, we have $\mathbf{Z}^{T} \widetilde{\mathbf{K}}^{-1} \mathbf{N}^{T} \mathbf{x}=\mathbb{W}^{M^{T}} \mathbf{A} \mathbf{x}(\lambda+1) / 2$. Since assuming $\mathbf{x} \in \operatorname{Ker}\left(\mathbf{P}^{T}\right)$ is equivalent to $\mathbb{W}^{M^{T}} \mathbf{A} \mathbf{x}=0$, the low-rank part of the above development vanishes when applied to $\mathbf{x}$ and it only remains that:

$$
\frac{\mathbf{M}_{3}+\mathbf{I}}{2} \mathbf{x}=\mathbf{H N} \widetilde{\mathbf{K}}^{-1} \mathbf{N}^{T} \mathbf{x}=\frac{\lambda+1}{2} \mathbf{x}
$$


We can now try to derive a coarse space which would warranty a given rate of convergence $0<\rho<1$. We can split the spectrum in two parts: superscript $M$ (macro) gathers the bad eigenvalues and associated eigenvectors. Bad eigenvalues are the ones responsible for poor convergence, that is to say eigenvalues whose absolute value is greater than $\rho$. Superscript $m$ (micro) is used for the rest of the spectrum.

In order to remove the bad spectrum from the resolution with the multiscale constraint, we would need to find a macro subspace $\mathbb{W}^{M}$ such that:

$$
\operatorname{Span}\left(\mathbf{X}^{M}\right) \subset \operatorname{Range}\left(\mathbf{P}^{T}\right)=\operatorname{Span}\left(\mathbf{A}^{T} \mathbb{W}^{M}\right)
$$

Unfortunately, this cannot be achieved. Indeed, it is possible to write $\mathbf{X}^{M}$ as $\mathbf{Q} \mathbf{A}^{T} \mathbf{X}_{A}^{M}+\mathbf{B}^{T} \mathbf{X}_{B}^{M}$, and the $\mathbf{X}_{B}^{M}$ can not be affected. The best we can do is to remove the $\mathbf{X}_{A}^{M}$ part, for a well-chosen orthogonality Q.

Proposition 9 (Quasi-optimal macro constraint). In view of previous analysis, choosing $\mathbf{Q}=\mathbf{H}$ to define $\mathbf{X}_{A}^{M}$, we propose to define the macro constraint as:

$$
\mathbb{W}^{M}=\left(\mathbf{A H A}^{T}\right)^{-1} \mathbf{A H N Y} \mathbf{Y}^{M}
$$

This choice leads to $\operatorname{Span}\left(\mathbf{A}^{T} \mathbb{W}^{M}\right)=\operatorname{Span}\left(\mathbf{A}^{T} \mathbf{X}_{A}^{M}\right)$.

Proof. Due to the relation between $\mathbf{Y}^{M}$ and $\mathbf{X}^{M}$ the previous equation is equivalent to:

$$
\begin{aligned}
\mathbb{W}^{M} & =\left(\mathbf{A H A}^{T}\right)^{-1} \mathbf{A} \underbrace{\mathbf{H N}\left(\mathbf{K}+\mathbf{N}^{T} \mathbf{H N}\right)^{-1} \mathbf{N}^{T} \mathbf{X}^{M}}_{\mathbf{X}^{M} \frac{\boldsymbol{\Lambda}+1}{2} \text { with eq } 30} \\
& =\left(\mathbf{A H A}^{T}\right)^{-1} \mathbf{A} \mathbf{X}^{M} \frac{\boldsymbol{\Lambda}+1}{2}
\end{aligned}
$$

Writing $\mathbf{X}^{M}=\mathbf{H} \mathbf{A}^{T} \mathbf{X}_{A}^{M}+\mathbf{B}^{T} \mathbf{X}_{B}^{M}$, the expression of $\mathbb{W}^{M}$ becomes:

$$
\begin{aligned}
\mathbb{W}^{M} & =\left(\mathbf{A} \mathbf{H} \mathbf{A}^{T}\right)^{-1}(\mathbf{A} \mathbf{H} \mathbf{A}^{T} \mathbf{X}_{A}^{M}+\underbrace{\mathbf{A} \mathbf{B}^{T}}_{=0} \mathbf{X}_{B}^{M}) \frac{\boldsymbol{\Lambda}+1}{2} \\
& =\mathbf{X}_{A}^{M} \frac{\boldsymbol{\Lambda}+1}{2}
\end{aligned}
$$

Remark 4 (Difference with usual macrospace). Contrarily to the usual macrospace, here the modes are originating from the subdomains and then projected on the interfaces, instead of directly emanating from the interfaces. This has several consequences:

- With our method, one subdomain's mode has components on each interface of that subdomain, whereas one interface receives information from the two neighbors it links.

- The dimension of the coarse problem may strongly differ. Considering a cube with $N$ subdomains per side, there are $N^{3}$ subdomains and $3 N^{2}(N-1)$ interfaces, assuming the minimal rigid-body motions macro-modes, our coarse space has $6 N^{3}$ columns compared to the $\simeq 18 N^{3}$ columns of the usual method.

- With our method, higher order modes are built based on spectral considerations on subdomains, where each subdomain adapts the number of its contributions to a given criterion. In the standard method, a common higher degree is used to define the macro kinematics of the interfaces, in general regardless of the heterogeneity. 


\section{$7 \quad$ Numerical assessments}

The following three test-cases permit to assess the performance of the multiscale Latin method, considering the proposed search directions and the new macrospace. For practical reasons, the generalized eigenvalue problem at the boundary of subdomains 40 is defined as:

$$
\begin{aligned}
\left(\mathbf{K}+\mathbf{N}^{T} \mathbf{H N}\right) \mathbf{Y} & =\breve{\lambda}\left(\mathbf{N}^{T} \mathbf{H N}\right) \mathbf{Y} \\
\text { or in condensed form: }\left(\mathbf{S}+\mathbf{N}_{b} \mathbf{H} \mathbf{N}_{b}^{T}\right) \mathbf{Y} & =\breve{\lambda}\left(\mathbf{N}_{b}^{T} \mathbf{H} \mathbf{N}_{b}\right) \mathbf{Y}
\end{aligned}
$$

These eigenvalues are the inverses of the ones used previously: $\breve{\lambda}=1 / \lambda \geqslant 1$, and the smaller $\min \left(\breve{\lambda}_{i}\right)$, the worse the rate of convergence is. When using the spectrum-based coarse space, we will select all modes associated with eigenvalues $1 \leqslant \breve{\lambda}_{i} \leqslant \breve{\rho}$, where $\breve{\rho}$ is a user parameter.

We remind the three variants of the search direction that we introduced:

- $\mathbf{H}_{O}=\operatorname{diag}\left(\widetilde{\mathbf{N}}_{b} \widetilde{\mathbf{S}} \widetilde{\mathbf{N}}_{b}^{T}\right)$ is the search direction presented in Proposition 4 with $\widetilde{\mathbf{S}}$ the superlumped stiffness matrix.

- $\mathbf{H}_{K}=\mathbf{A}^{T}\left(\mathbf{A D A}^{T}\right)^{-1} \mathbf{A}$ with $\mathbf{D}=\widetilde{\mathbf{N}}_{b} \operatorname{diag}\left(\mathbf{K}_{b b}\right)^{-1} \widetilde{\mathbf{N}}_{b}^{T}$.

- $\mathbf{H}_{S}=\mathbf{A}^{T}\left(\mathbf{A D A}^{T}\right)^{-1} \mathbf{A D} \widetilde{\mathbf{N}}_{b} \operatorname{diag}(\mathbf{S})^{-1} \widetilde{\mathbf{N}}_{b}^{T} \mathbf{D A}^{T}\left(\mathbf{A D} \mathbf{A}^{T}\right)^{-1} \mathbf{A}$.

\subsection{Chessboard test case}

We propose a test-case similar to the one from [15] representing a heterogeneous chessboard subjected to gravity and clamped one its bottom face. The objectives are:

1. To conduct a weak scalability study in a case where partition resolves the heterogeneity, and to compare the $\mathbf{H}_{O}$ and $\mathbf{H}_{S}$ search directions.

2. To conduct a strong scalability study with partitions that involves heterogeneous subdomains, and to compare the $\mathbf{H}_{S}$ and $\mathbf{H}_{K}$ search directions.

Whatever the studied case, the materials are linear isotropic elastic (see Table 1). The heterogeneity comes from the distinct material between 2 neighbored-squares of the chessboard.

\begin{tabular}{cc} 
material 1 & material 2 \\
\hline$E_{1}=200000 \mathrm{MPa}$ & $E_{2}=2 \mathrm{MPa}$ \\
$\nu_{1}=0.3$ & $\nu_{2}=0.3$ \\
\hline
\end{tabular}

Table 1: Description of the materials (chessboard test case)

\subsubsection{Weak scalability with partitions resolving the heterogeneity}

In this part we study the case where partitions resolve the heterogeneity. It means that a subdomain is composed of one only material. Four structures with 16, 36, 64 and 256 subdomains respectively are simulated under a gravity load (Figure 3). We choose a criterion of $\breve{\rho}=1.1$ in order to select the spectral modes.

Comparison of $\mathbf{H}_{O}$ and $\mathbf{H}_{S}$ In Figure 4 , the convergence and the spectrum of subdomains for these two kinds of search directions are compared. Several comments can be made:

- Figure 4a: with the search direction $\mathbf{H}_{O}$, a dependency of the convergence rate is observed with respect to the number of subdomains. On the contrary with the search direction $\mathbf{H}_{S}$, the convergence is more stable and asymptotically faster. 


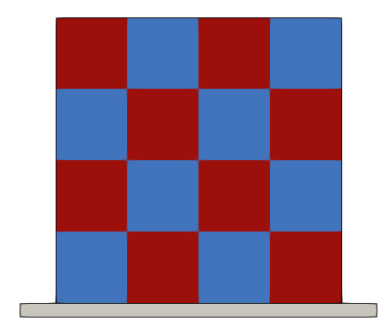

(a) 16 subdomains

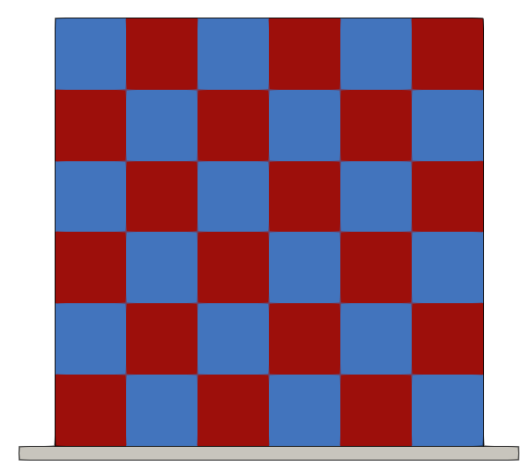

(b) 36 subdomains

Figure 3: Different partitions resolving heterogeneities.

- Figure 4b; we focus on the spectrum of soft and stiff subdomains for both search directions. Contrary to the results obtained by [15], in the $\mathbf{H}_{O}$ case, the subdomains do not only select the rigid body modes, but also include a few more modes due to our mixed approach. However, in the $\mathbf{H}_{S}$ case, the rigid subdomains include only the rigid body modes and a huge jump is observed in the spectrum.

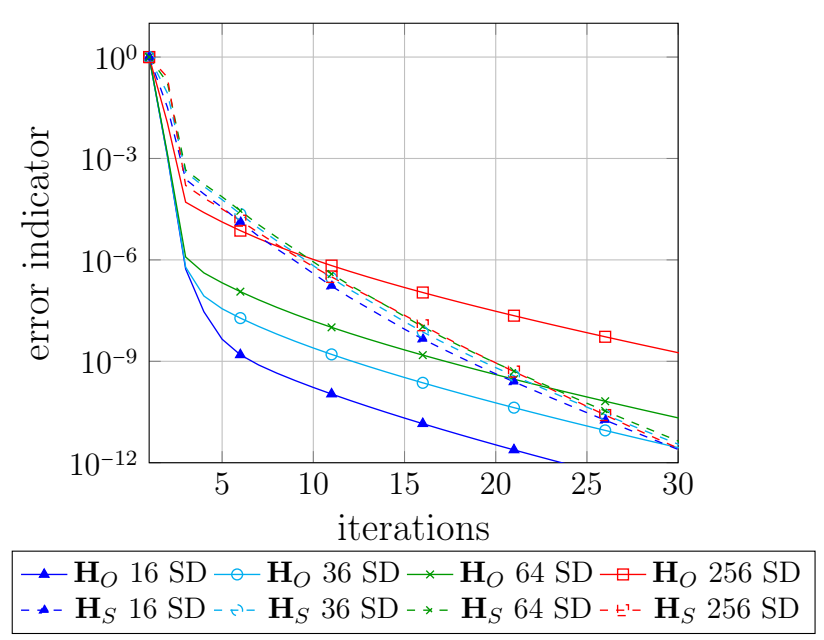

(a) Convergence rate

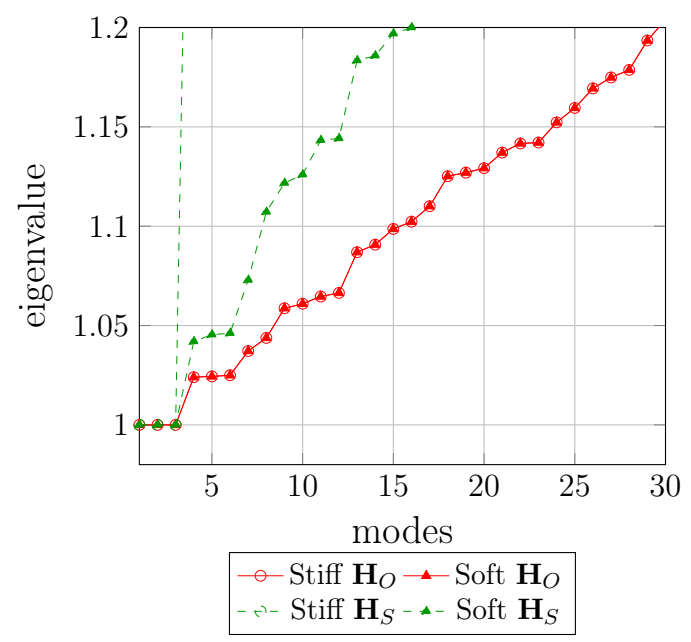

(b) Spectrum of stiff and soft subdomains

Figure 4: Weak scalability - effect of the search direction $\mathbf{H}_{O}$ and $\mathbf{H}_{S}$ (chessboard test case). 
Comparison with the standard multiscale approach Henceforth, we choose the $\mathbf{H}_{S}$ search direction in order to compare the spectral approach with the standard one. We observe in Figure 5 that the convergence rate remains similar for both procedures. Indeed, in this case where the partition resolves the heterogeneity, the spectral approach only selects the rigid body and simple deformation modes (see Figure 6) which are similar to the ones chosen in the standard approach. For the standard approach, 6 modes are defined per interface.

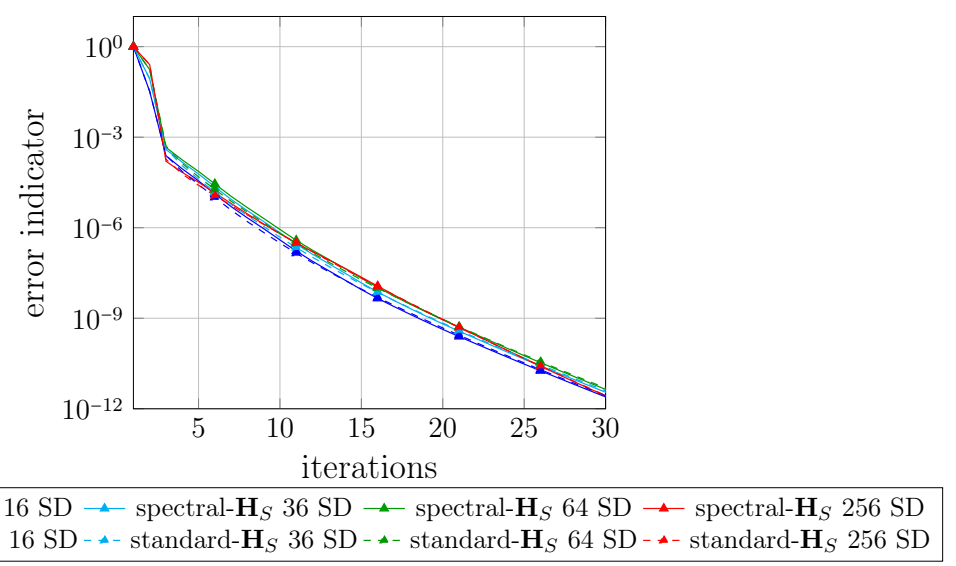

Figure 5: Weak scalability - convergence rate for the standard and spectral approaches with different number of subdomains (chessboard test-case).

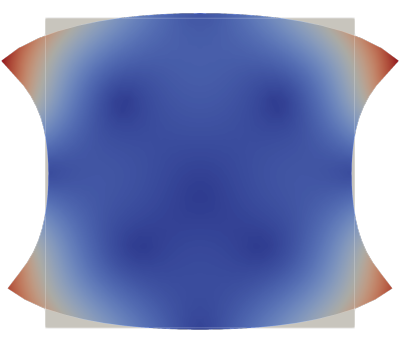

(a) $\breve{\lambda}_{3}=1.042$

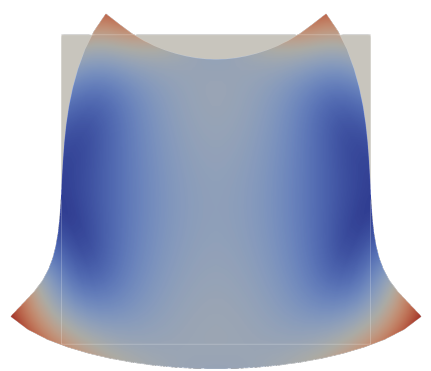

(c) $\breve{\lambda}_{5}=1.046$

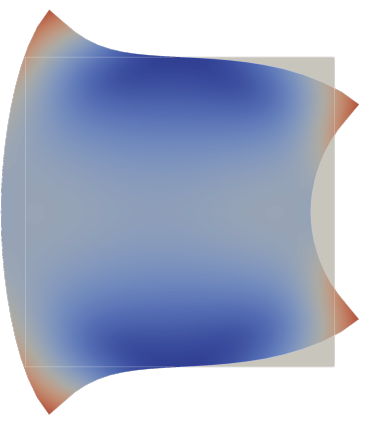

(b) $\breve{\lambda}_{4}=1.045$

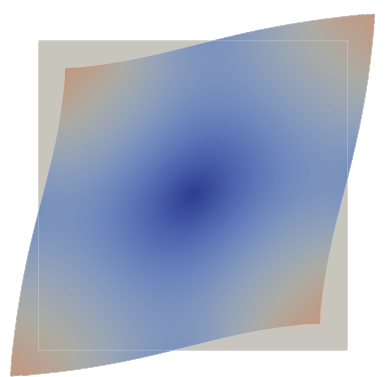

(d) $\breve{\lambda}_{6}=1.073$

Figure 6: Weak scalability - additional modes for soft subdomains (chessboard test case). 


\subsubsection{Strong scalability}

Now we study the strong scalability of the methods by keeping fixed the number of degrees of freedom and modifying the partition. For this, we start from the previous 256-subdomain case and we regroup subdomains four by four to create a new partition of $8 \times 8$ new subdomains. Therefore, these new subdomains are no more homogeneous, and they are composed of the two materials in a $2 \times 2$ chessboard scheme. From this new partition we apply the same procedure to obtain a new $4 \times 4$ partition. Moreover - as the subdomains are heterogeneous - we use a $\breve{\rho}=1.2$ criterion to select the spectral modes with a maximum of 30 modes selected per subdomain. Several comments can be made:

- Figure 7a 7b with heterogeneous subdomains the standard modes are no more able to represent the heterogeneity correctly. Thus, the more heterogeneous the subdomains are, the worse the convergence rate is. In particular, the convergence of the $4 \times 4$ partition leads to stagnation whereas the $8 \times 8$ one keeps converging with a few oscillations. On the other side, the spectral approach is not really disrupted as it selects the most adapted modes automatically, however the size of the coarse problem increases with the number of modes. Moreover, when using $\mathbf{H}_{S}$ we can observe that the convergence rate is extremely similar for the $4 \times 4$ and $8 \times 8$ partitions, whereas the $16 \times 16$ partition (which resolves the heterogeneity) has an inferior performance. In the case of $\mathbf{H}_{K}$, we note an improvement for the $8 \times 8$ and $16 \times 16$ respect to the $4 \times 4$ partition.

- Figure $7 \mathrm{c}-7 \mathrm{~d}$, it is observed that the more heterogeneous the subdomains are, the lower the first eigenvalues are. Therefore, more modes are selected. We also notice that the $\mathbf{H}_{K}$ case with 16 subdomains needs more than 30 modes to satisfy the criterion $\breve{\rho}=1.2$ and represent the whole subspace. On the other side, for the three partitions more modes are selected for $\mathbf{H}_{K}$ than for $\mathbf{H}_{S}$ respectively, explaining why $\mathbf{H}_{K}$ leads to faster convergence for each partition.

- Figure 8, the first modes for a subdomain belonging to the $4 \times 4$ partition are shown, where we can observe the role of the softer material in these deformations.

\section{$7.22 \mathrm{D}$ heterogeneous beam}

In this example we show the performance of the method when the heterogeneity pattern is more complex and where the interfaces see crossing and non-crossing heterogeneity, as illustrated in Figure 9. The studied structure consists of a 2D beam with the material pattern described in Figure 10 and Table 2, submitted to a vertical flexion load on its right end. The 4-subdomain pattern is repeated 16 times to obtain 64 subdomains in total.

\begin{tabular}{cccc} 
material 0 & material 1 & material 2 & material 3 \\
\hline$E_{0}=2 \mathrm{MPa}$ & $E_{1}=200000 \mathrm{MPa}$ & $E_{2}=2000 \mathrm{MPa}$ & $E_{3}=50000 \mathrm{MPa}$ \\
$\nu_{0}=0.3$ & $\nu_{1}=0.49$ & $\nu_{2}=0.3$ & $\nu_{3}=0.3$ \\
\hline
\end{tabular}

Table 2: Description of the materials (2D heterogeneous test case)

\subsubsection{Study of the spectrum}

We show the spectrum of the first five subdomains in Figure 11. Excepted for the first subdomain, all others have a similar spectrum: three rigid body modes associated with the triple eigenvalue 1 followed by six modes with eigenvalues close to 1 . Then, a gap is observed in the spectrum and no more modes are selected as we cut at $\breve{\rho}=1.05$. One can remark that after the ninth first modes, the spectrum for $\mathbf{H}_{K}$ is lower than for $\mathbf{H}_{S}$. We illustrate the first nine modes in Figure 12 concerning the third subdomain. Regarding the first subdomain, no rigid body modes are selected since it is a clamped subdomain. A few modes are selected, they are shown in Figure 13. 


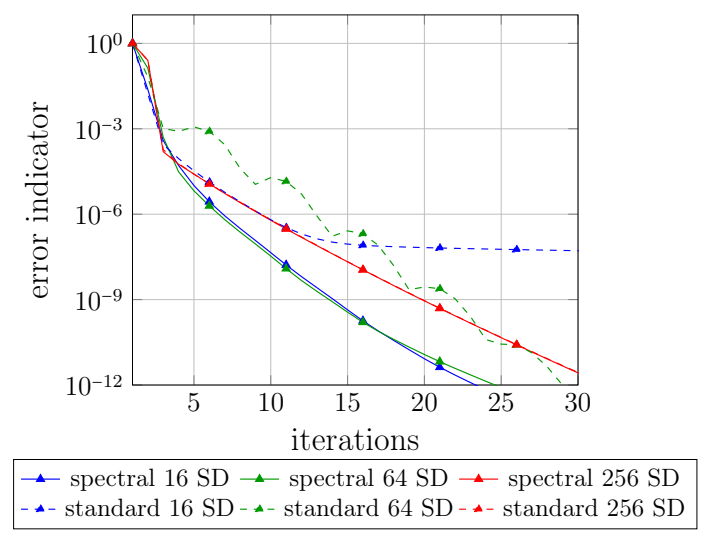

(a) convergence rate for $\mathbf{H}_{S}$

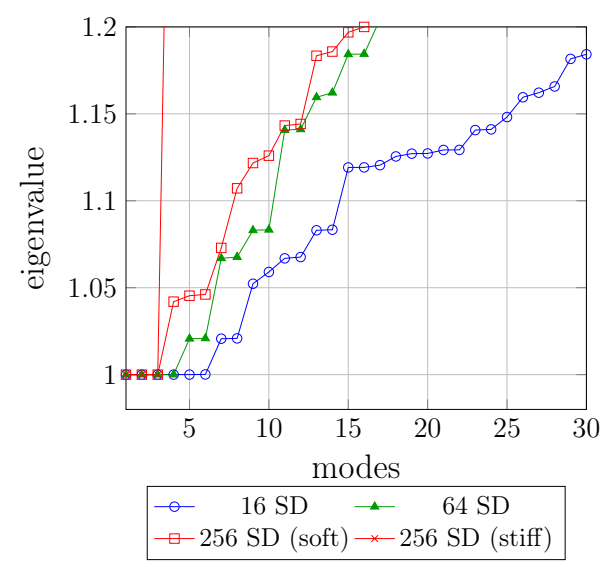

(c) spectrum for $\mathbf{H}_{S}$

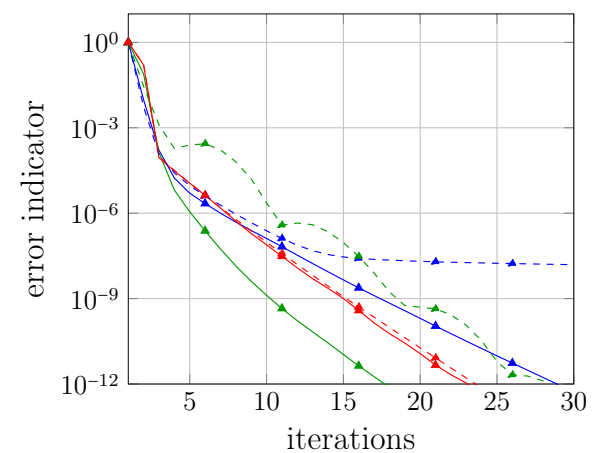

$\longleftarrow$ spectral $16 \mathrm{SD} \_$spectral $64 \mathrm{SD} \_$spectral $256 \mathrm{SD}$ - $\_$- standard $16 \mathrm{SD}$ - $\_$- standard $64 \mathrm{SD}$ - $\_$- standard $256 \mathrm{SD}$

(b) convergence rate for $\mathbf{H}_{K}$

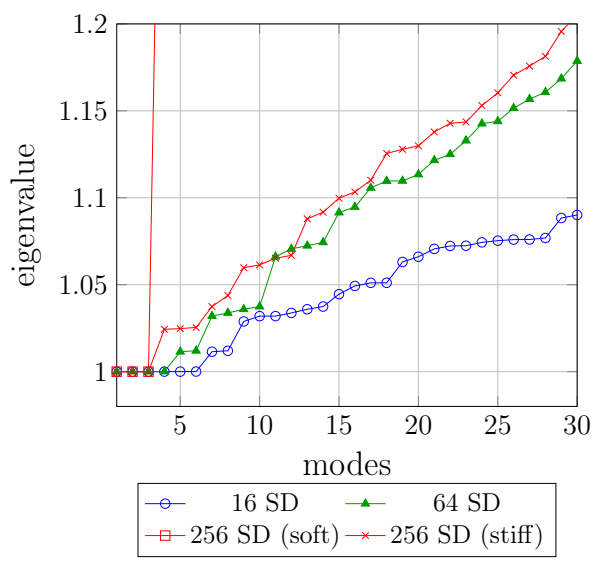

(d) spectrum for $\mathbf{H}_{K}$

Figure 7: Strong scalability - effect of the search direction $\mathbf{H}_{S}$ and $\mathbf{H}_{K}$ (chessboard test case).

\subsubsection{Comparison with the standard multiscale approach}

We contrast the convergence ratio of the spectral and classical coarse spaces. The Figure 14 shows the evolution of iterations for the two approaches and also that of the monoscale. We note a tremendous improvement with the spectral macrospace. Moreover, the convergence ratio depends on the search direction $\left(\mathbf{H}_{S}\right.$ or $\left.\mathbf{H}_{K}\right)$.

\subsection{A 3D heterogeneous test case}

We are here focused on a 3D cubic structure partitioned into 64 subdomains $(4 \times 4 \times 4)$ with heterogeneity both inside subdomains and crossing interfaces at corners, as detailed in Figure 15. The difficulty lies in the stiffness contrast (see Table 3) between the very soft matrix - material 0 - compared to the stiff inclusions - materials 1 and 2 - which leads to a very bad conditioning, especially due to the corner inclusions. For this example, only the search direction $\mathbf{H}_{K}$ is used.

\begin{tabular}{ccc} 
material 0 & material 1 & material 2 \\
\hline$E_{0}=2 \mathrm{MPa}$ & $E_{1}=200000 \mathrm{MPa}$ & $E_{2}=200000 \mathrm{MPa}$ \\
$\nu_{0}=0.3$ & $\nu_{1}=0.3$ & $\nu_{2}=0.3$ \\
\hline
\end{tabular}

Table 3: Description of the materials (3D heterogeneous test case). 


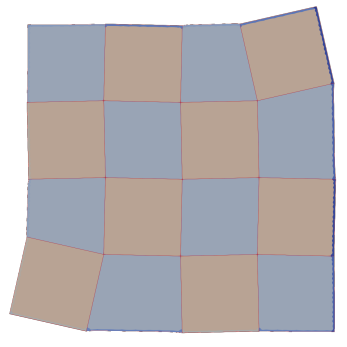

(a) $\breve{\lambda}_{3}=1.0000205$

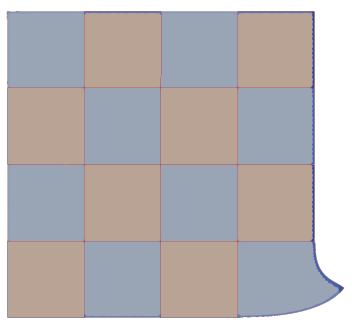

(e) $\breve{\lambda}_{7}=1.052$

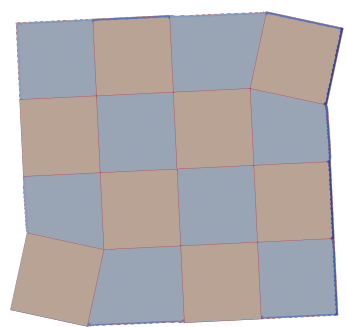

(b) $\breve{\lambda}_{4}=1.000025$

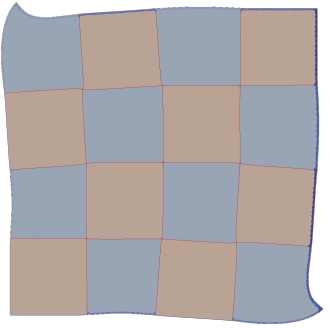

(f) $\breve{\lambda}_{8}=1.059$

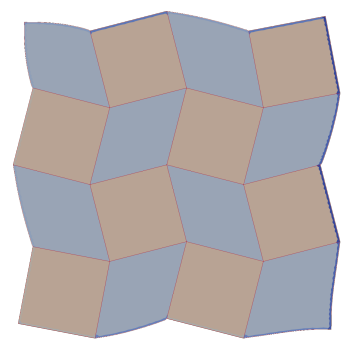

(c) $\breve{\lambda}_{5}=1.00012$

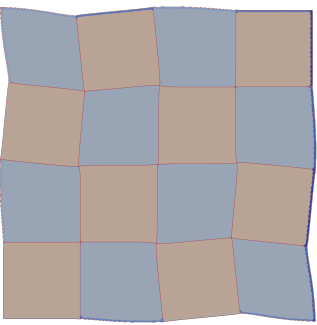

(g) $\breve{\lambda}_{9}=1.067$

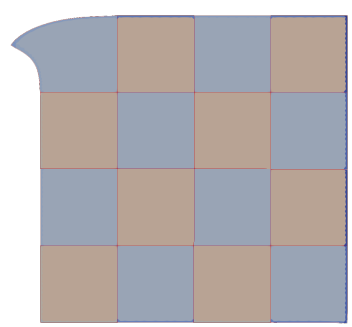

(d) $\breve{\lambda}_{6}=1.021$

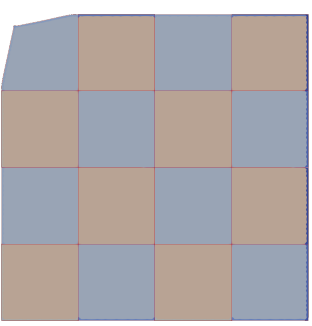

(h) $\breve{\lambda}_{10}=1.068$

Figure 8: Strong scalability - modes for an heterogeneous subdomain where the softer material is in bleu (chessboard test case).

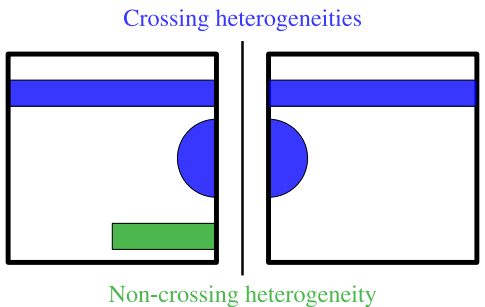

Figure 9: Example of crossing and non-crossing interfaces.

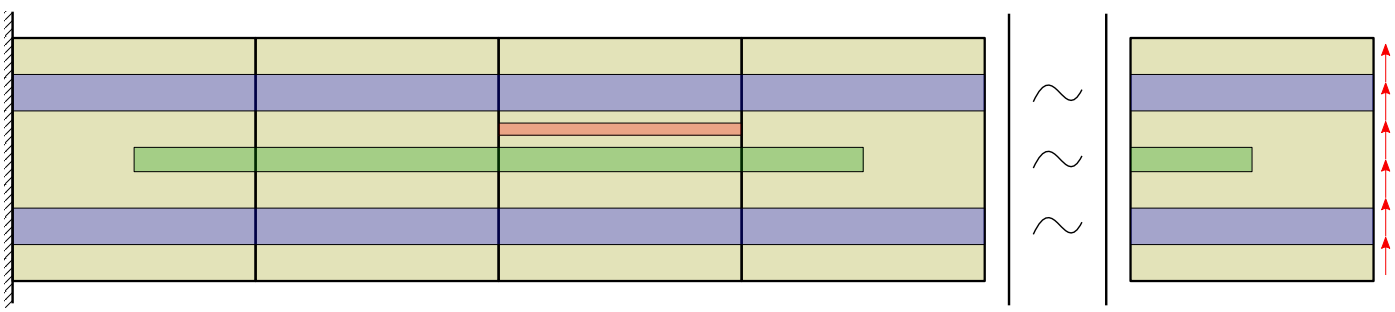

Figure 10: Pattern of materials - material 0, material 1, material 2, material 3.

\subsubsection{Homogeneous structure}

Firstly, we stay in a homogeneous case where the inclusions are made out of the same material as the matrix. The purpose is to expose that rigid body modes are not enough to obtain good performance.

The red plot in Figure 16 shows the spectrum of a subdomain located inside the structure, where we 


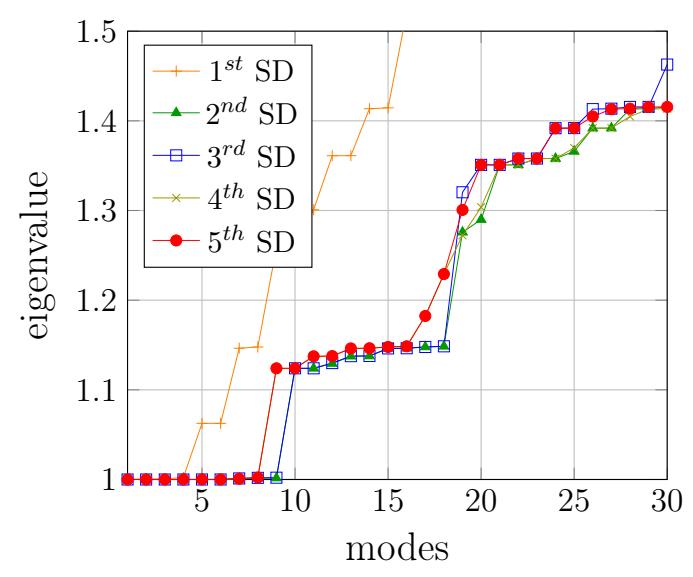

(a) spectrum for $\mathbf{H}_{S}$

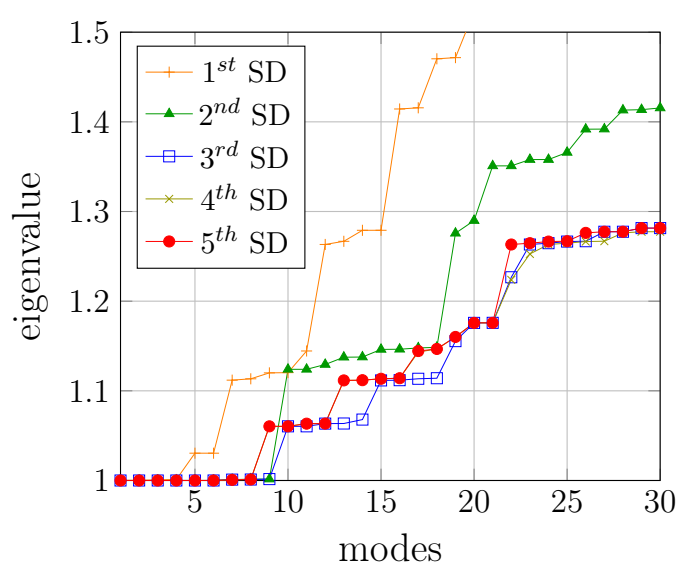

(b) spectrum for $\mathbf{H}_{K}$

Figure 11: Spectrum of the first five subdomains (2D heterogeneous test case).

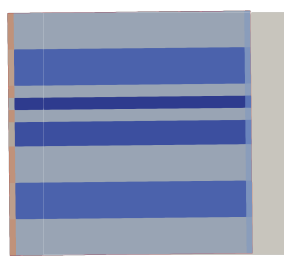

(a) $\breve{\lambda}_{0}=1$

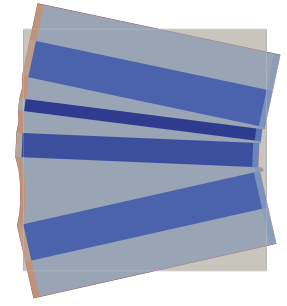

(d) $\breve{\lambda}_{3}=1.0000035$

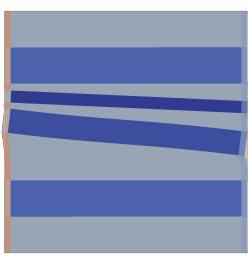

(g) $\breve{\lambda}_{6}=1.0012$

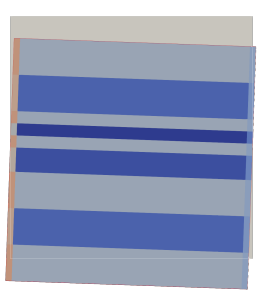

(b) $\breve{\lambda}_{1}=1$

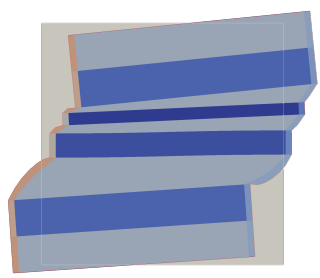

(e) $\breve{\lambda}_{4}=1.0000068$

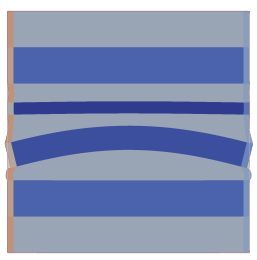

(h) $\breve{\lambda}_{7}=1.0018$

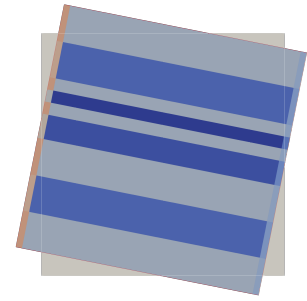

(c) $\breve{\lambda}_{2}=1$

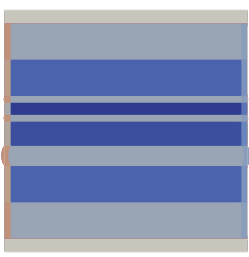

(f) $\breve{\lambda}_{5}=1.000011$

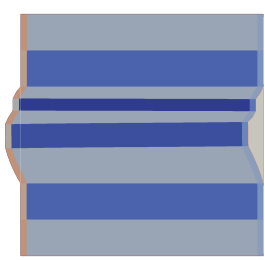

(i) $\breve{\lambda}_{8}=1.002$

Figure 12: First nine modes of the third subdomain and their associated eigenvalues (2D heterogeneous test case). 


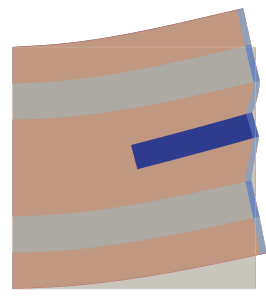

(a) $\breve{\lambda}_{0}=1.000223$

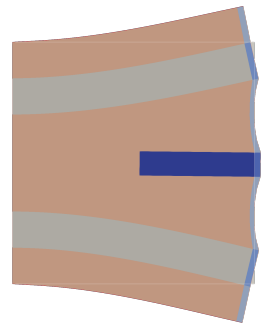

(b) $\breve{\lambda}_{1}=1.000228$

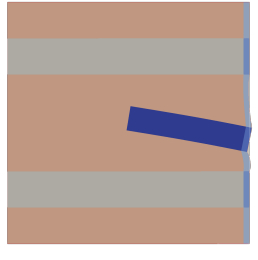

(c) $\breve{\lambda}_{2}=1.00085$

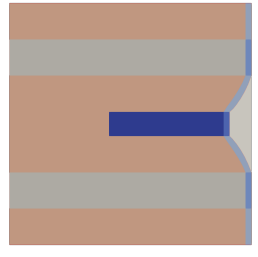

(d) $\breve{\lambda}_{3}=1.0022$

Figure 13: First four modes of the first subdomain and their associated eigenvalues (2D heterogeneous test case).

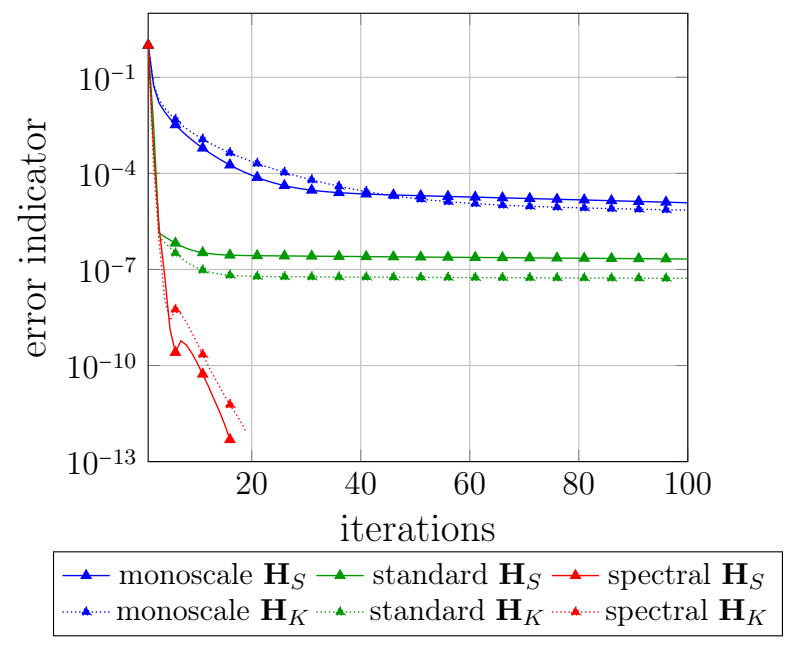

Figure 14: Convergence ratio for standard and spectral approaches (2D heterogeneous test case).

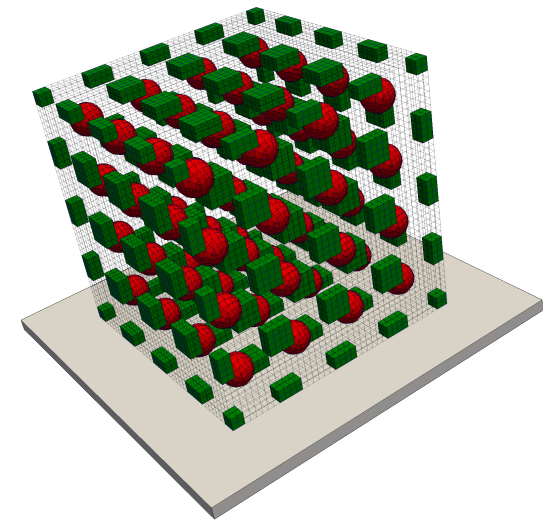

(a) the whole structure

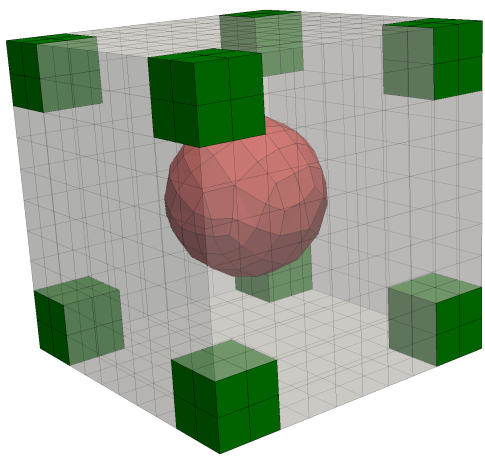

(b) a subdomain

Figure 15: Geometry, material heterogeneity and partition of the 3D heterogeneous structure (material 0, material 1 and material 2).

perceive some jumps, but they are not very strong. To illustrate the need of taking into account more than the very first modes, Figure $18 \mathrm{a}$ presents the convergence with the spectral approach for several criteria: 


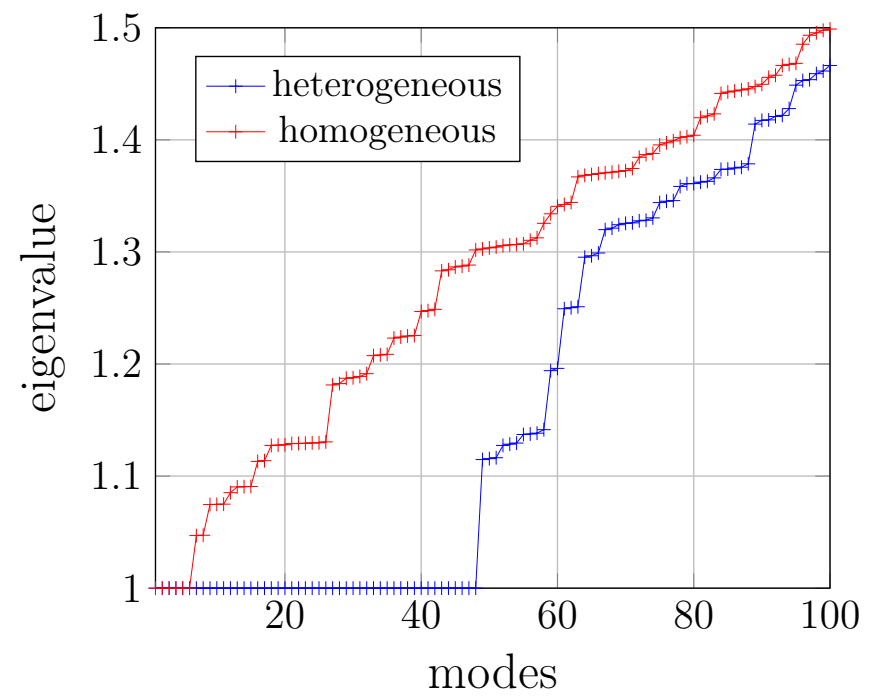

Figure 16: Spectrum of an internal subdomain (3D test case).

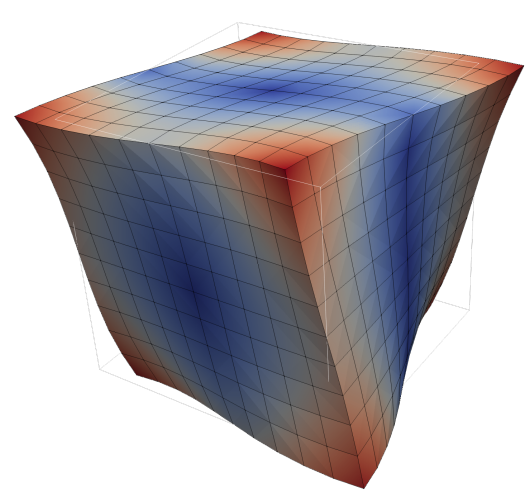

(a) $\breve{\lambda}_{6}=1.047$

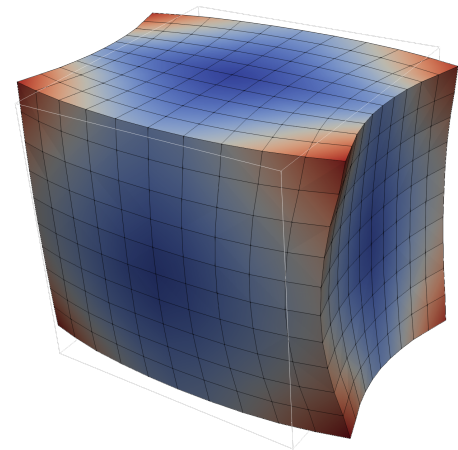

(b) $\breve{\lambda}_{15}=1.113$

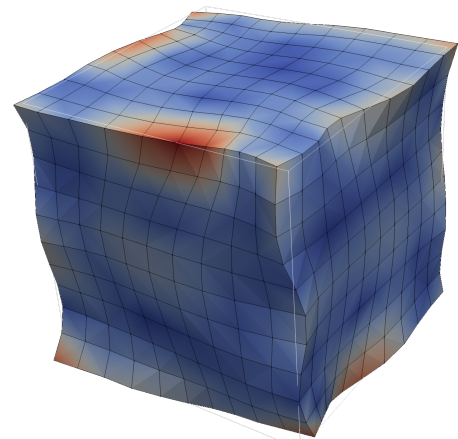

(c) $\breve{\lambda}_{75}=1.397$

Figure 17: Some modes and their associated eigenvalues (3D homogeneous test case).

from $\breve{\rho}=1.1$ to $\breve{\rho}=1.5$. The main conclusion is that the first modes containing combinations of rigid body motions and other simple deformations are not sufficient. Indeed, using a criterion of $\breve{\rho}=1.2$ the number of selected modes is doubled but it enables dividing by two the number of iterations to reach an error indicator of $10^{-13}$. Choosing a bit more modes with a criterion of $\breve{\rho}=1.3$, the convergence at the beginning is enhanced. However, it is not necessary to select more modes, because the convergence with criteria of $\breve{\rho}=1.3, \breve{\rho}=1.4$ and $\breve{\rho}=1.5$ remains very similar. Figure 17 shows some modes selected with different criteria. While we recognize simple deformation for the first ones, it becomes more erratic for the last ones and it remains very local. As confirmed in Figure 18a, these last modes do not really improve the convergence of the algorithm. Figure $18 \mathrm{~b}$ compares the standard approach considering only rigid body modes and simple interface deformations. Obviously rigid body motions (6 modes) are not able to capture all the suitable information. 


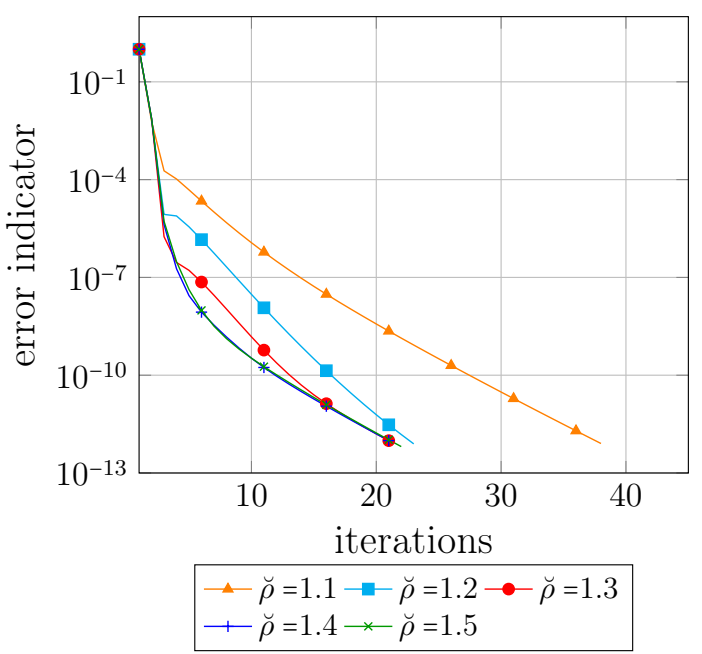

(a) spectral macrospace

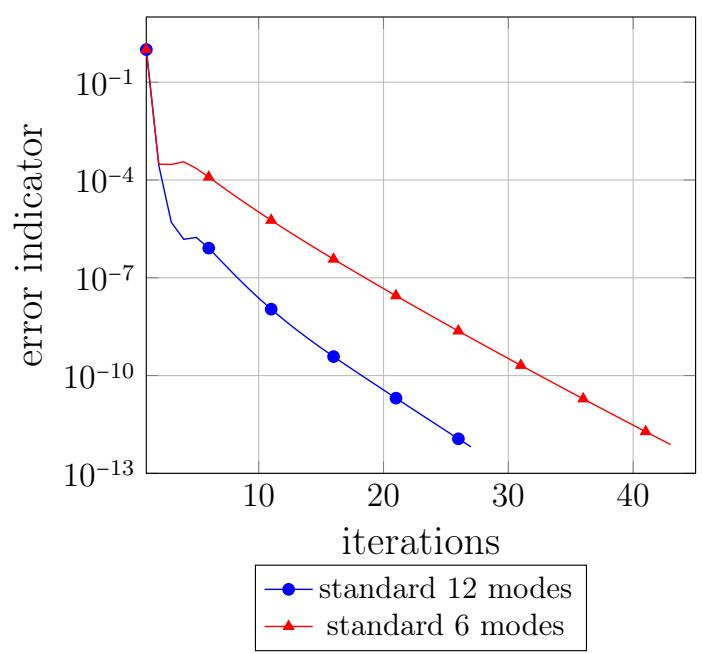

(b) standard macrospace

Figure 18: Convergence ratio for standard and spectral approaches (3D test case).

\subsubsection{Heterogeneous structure}

The 3D structure is here analyzed taking into account material parameters with a heterogeneity ratio of $10^{5}$ (see Table 3). The spectrum of an internal subdomain is blue plotted in Figure 16. The spectrum of the homogeneous case allows to emphasize that the heterogeneous case has a very flat spectrum near to 1 for the almost 50 first modes. These first modes are rigid body motions and simple deformations of the stiff inclusions. In Figure 19 a few modes are shown.

Figure 20 exposes what occurs if the initial plateau of the spectrum (eigenvalues close to 1) is partially taken into account. In fact, only 20 modes per subdomain are not satisfying; on the contrary even the weak criterion $\breve{\rho}=1.1$ is enough to capture the bad modes leading to a significantly improved convergence rate (fewer than 30 iterations to reach an error of $10^{-12}$ ). Selecting more modes by augmenting the criterion helps to improve the convergence: $\breve{\rho}=1.4$ seems to be most adapted in this case, whereas the more stringent $\breve{\rho}=1.5$ leads to selecting more than 100 modes per subdomains without accelerating.

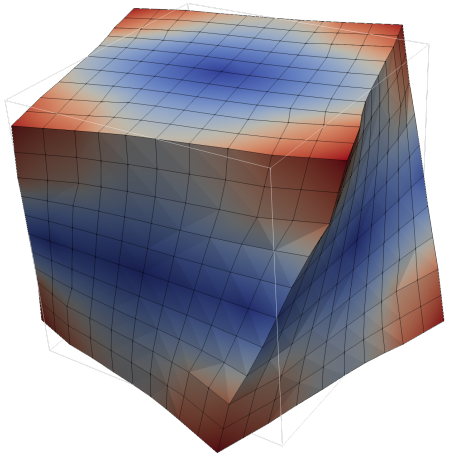

(a) $\breve{\lambda}_{6}=1.00000153$

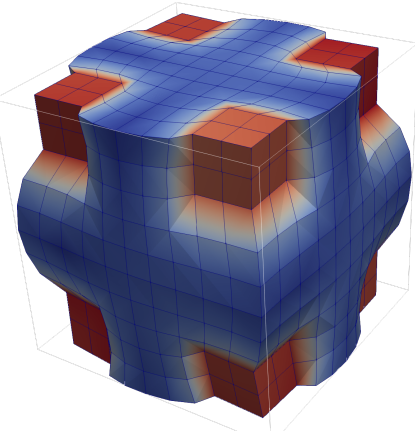

(b) $\breve{\lambda}_{30}=1.0000102$

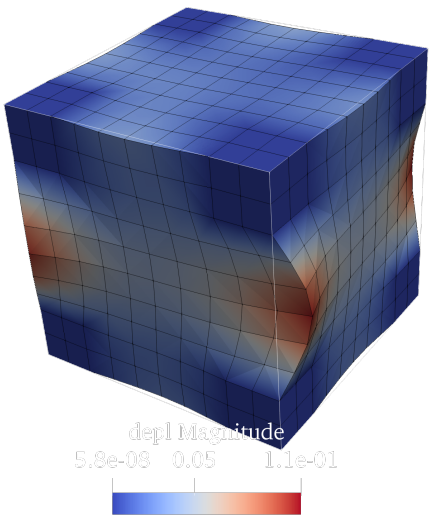

(c) $\breve{\lambda}_{48}=1.115$

Figure 19: Some modes and their associated eigenvalues (3D heterogeneous test case). 


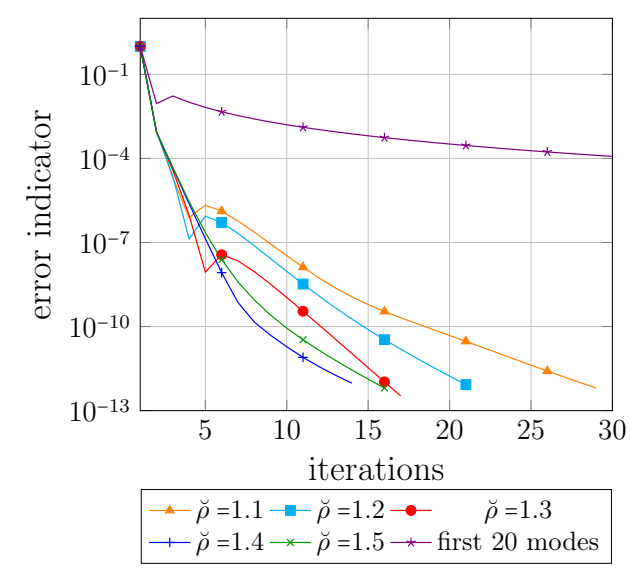

Figure 20: Convergence ratio for the spectral approach (3D heterogeneous test case).

\section{Conclusion}

This paper proposes an analysis of the mono and multiscale Latin method in the presence of perfect interfaces. It shows that the convergence rate of the monoscale method can be studied through local generalized eigenvalue problems. It is then possible to tell which spectral contributions are slowing down the convergence, and gather them in a "macrospace" used to constrain the resolution process. Unfortunately, in current state, with the multiscale Latin method only constraining the interface force field, it is not possible to warranty the bad part of the spectrum not being activated. Anyhow, even without strict bound on the convergence rate, it is possible to assess a new spectrum-motivated coarse space.

The performance of the method is illustrated of various severe heterogeneous examples. The first one consists in a weak and strong scalability study of a heterogeneous chessboard. The second test case is a $2 \mathrm{D}$ slender beam with various heterogeneity patterns. We observe very similar results respecto to the ones in [15] and we illustrate that the standard modes chosen for the Latin method are no more sufficient and modes adapted to the heterogeneity are required. The last test case is a 3D structure with spherical and cubic stiff inclusions into a softer material. We show that choosing more modes in addition to the rigid body modes is relevant, even in the homogeneous case, and compulsory in the heterogeneous case.

Future work will concern the full control of the spectrum of the multiscale operator, probably using more complex constraint, also applied to the displacement field.

\section{Acknowledgment}

This work was partly funded by the international project ECOS-CONICYT C17E04 between LMT and Universidad de Talca, and by the French project PIA PAMSIM.

\section{References}

[1] V. Dolean, P. Jolivet, F. Nataf, An introduction to domain decomposition methods: algorithms, theory, and parallel implementation, SIAM, 2015.

[2] J. Mandel, B. Sousedík, Adaptive selection of face coarse degrees of freedom in the BDDC and the FETI-DP iterative substructuring methods, Comput. Methods Appl. Mech. Eng. 196 (8) (2007) 1389-1399. doi:10.1016/j.cma.2006.03.010.

[3] J. Mandel, B. Sousedík, J. Šístek, Adaptive BDDC in three dimensions, Math. Comput. Simul. 82 (10) (2012) 1812-1831. arXiv:arXiv:0910.5863v2, doi:10.1016/j.matcom.2011.03.014. 
[4] A. Klawonn, M. Kühn, O. Rheinbach, Adaptative coarse space for FETI-DP in three dimensions, SIAM J. SCI. Comput. 38 (5) (2016) A2880-A2911. doi:10.1137/15M10496101.

[5] A. Klawonn, P. Radtke, O. Rheinbach, A comparison of adaptive coarse spaces for iterative substructuring in two dimensions, Electron. Trans. Numer. Anal. 45 (2016) 75-106.

[6] C. Pechstein, C. R. Dohrmann, A unified framework for adaptive BDDC, Electronic Transactions on Numerical Analysis 46 (2017) 273-336.

[7] A. Klawonn, M. Kühn, O. Rheinbach, Adaptative FETI-DP and BDDC methods with a generalized transformation of basis for heterogeneous problems, Electronic Transactions on Numerical Analysis 49 (2018) 1-27. doi:10.1553/etna.

[8] A. Klawonn, M. Kühn, O. Rheinbach, Coarse spaces for FETI-DP and BDDC methods for heterogeneous problems: connections of deflation and a generalized transformation-of-basis approach, Electronic Transactions on Numerical Analysis 52 (2020) 43-76. doi:10.1553/etna.

[9] M. J. Gander, Optimized Schwarz Methods, SIAM Rev. 44 (2) (2006) 699-731. doi:10.1137/ S0036142903425409.

[10] A. St-Cyr, M. J. Gander, S. J. Thomas, Optimized Multiplicative, Additive, and Restricted Additive Schwarz Preconditioning, SIAM Journal on Scientific Computing 29 (6) (2007) 2402-2425. arXiv: arXiv:1011.1669v3, doi:10.1137/060652610. URL http://epubs.siam.org/doi/10.1137/060652610

[11] M. J. Gander, Schwarz methods over the course of time, Electronic Transactions on Numerical Analysis 31 (5) (2008) 228-255. doi:10.1007/s00595-010-4443-5.

[12] M. J. Gander, Y. Xu, Optimized Schwarz methods for model problems with continuously vaiable coefficients, SIAM Journal of Scientific Computing 38 (5) (2016) A2964-A2986. doi:10.1137/15M1053943. URL epubs . siam.org/doi/abs/10.1137/15M1053943

[13] M. J. Gander, T. Vanzan, Heterogeneous optimized Schwarz methods for second order elliptic PDEs, SIAM Journal of Scientific Computing 41 (4) (2019) 2329-2354. doi:10.1137/18M122114X. URL epubs . siam.org/doi/abs/10.1137/18M122114X

[14] N. Spillane, V. Dolean, P. Hauret, F. Nataf, C. Pechstein, R. Scheichl, A robust two-level domain decomposition preconditioner for systems of PDEs, Comptes Rendus Math. 349 (23-24) (2011) 12551259. doi:10.1016/j.crma.2011.10.021.

[15] N. Spillane, D. J. Rixen, Automatic spectral coarse spaces for robust finite element tearing and interconnecting and balanced domain decomposition algorithms, Int. J. Numer. Methods Eng. 95 (11) (2013) 953-990. arXiv:1010.1724, doi:10.1002/nme.4534.

[16] N. Spillane, V. Dolean, P. Hauret, F. Nataf, C. Pechstein, R. Scheichl, Abstract robust coarse spaces for systems of PDEs via generalized eigenproblems in the overlaps, Numer. Math. 126 (4) (2014) 741-770. doi:10.1007/s00211-013-0576-y.

[17] F. Nataf, Mathematical analysis of robustness of two-level domain decomposition methods with respect to inexact coarse solves, Numerische Mathematik 144 (4) (2020) 811-833. doi:10.1007/ s00211-020-01102-6.

URL https://doi.org/10.1007/s00211-020-01102-6

[18] R. Haferssas, P. Jolivet, F. Nataf, A robust coarse space for Optimized Schwarz methodsSORASGenEO-2, Comptes Rendus Mathematique 353 (10) (2015) 959,963. doi:10.1016/j.crma.2015.07. 014 . 
[19] R. Haferssas, P. Jolivet, F. Nataf, An additive schwarz method type theory for lions's algorithm and a symmetrized optimized restricted additive schwarz method, SIAM Journal on Scientific Computing 39 (4) (2017) A1345-A1365. arXiv:https://doi.org/10.1137/16M1060066, doi:10.1137/16M1060066. URL https://doi.org/10.1137/16M1060066

[20] R. Bridson, C. Greif, A Multipreconditioned Conjugate Gradient Algorithm, SIAM J. Matrix Anal. Appl. 27 (4) (2006) 1056-1068. doi:10.1137/040620047.

[21] P. Gosselet, D. J. Rixen, F.-X. Roux, N. Spillane, Simultaneous FETI and block FETI: Robust domain decomposition with multiple search directions, Int. J. Numer. Methods Eng. 104 (10) (2015) 905-927. arXiv:1010.1724, doi:10.1002/nme.4946.

[22] N. Spillane, An Adaptive MultiPreconditioned Conjugate Gradient Algorithm, SIAM J. Sci. Comput. 38 (3) (2016) A1896-A1918. doi:10.1137/15M1028534.

[23] C. Bovet, A. Parret-Fréaud, N. Spillane, P. Gosselet, Adaptive multipreconditioned FETI: Scalability results and robustness assessment, Comput. Struct. 193 (2017) 1-20. doi:10.1016/J.COMPSTRUC. 2017.07.010.

[24] M. C. Leistner, P. Gosselet, D. J. Rixen, Recycling of Solution Spaces in Multi-Preconditioned FETI Methods Applied to Structural Dynamics, International Journal for Numerical Methods in Engineering 116 (2) (2018) 141-160. doi:10.1002/nme.5918.

[25] P. Ladevèze, Nonlinear computational structural mechanics: new approaches and non-incremental methods of calculation, Springer-Verlag New York, 1999. doi:10.1007/978-1-4612-1432-8.

[26] P. Ladevèze, A. Nouy, On a multiscale computational strategy with time and space homogenization for structural mechanics, Computer Methods in Applied Mechanics and Engineering 192 (28) (2003) 3061-3087, multiscale Computational Mechanics for Materials and Structures. doi:https://doi. org/10.1016/S0045-7825(03)00341-4.

URL http://www.sciencedirect.com/science/article/pii/S0045782503003414

[27] V. Roulet, L. Champaney, P.-A. Boucard, A parallel strategy for the multiparametric analysis of structures with large contact and friction surfaces, Advances in Engineering Software 42 (6) (2011) $347-358$.

[28] P. A. Guidault, O. Allix, L. Champaney, C. Cornuault, A multiscale extended finite element method for crack propagation, Computer Methods in Applied Mechanics and Engineering 197 (5) (2008) 381-399.

[29] P. Kerfriden, O. Allix, P. Gosselet, A three-scale domain decomposition method for the 3D analysis of debonding in laminates, Computational Mechanics 44 (3) (2009) 343-362.

[30] V. Visseq, P. Alart, D. Dureisseix, Towards an augmented domain decomposition method for nonsmooth contact dynamics models, Computational Particle Mechanics 1 (1) (2014) 15-26. doi: 10.1007/s40571-014-0005-8.

[31] A. Giacoma, D. Dureisseix, A. Gravouil, M. Rochette, Toward an optimal a priori reduced basis strategy for frictional contact problems with LATIN solver, Computer Methods in Applied Mechanics and Engineering 283 (2015) 1357-1381. doi:10.1016/j.cma.2014.09.005.

URL http://dx.doi.org/10.1016/j.cma.2014.09.005 
[32] P. Ladevèze, J.-C. Passieux, D. Néron, The latin multiscale computational method and the proper generalized decomposition, Computer Methods in Applied Mechanics and Engineering 199 (21) (2010) 1287 - 1296, multiscale Models and Mathematical Aspects in Solid and Fluid Mechanics. doi:https: //doi.org/10.1016/j.cma.2009.06.023. URL http://www.sciencedirect.com/science/article/pii/S0045782509002643

[33] N. Relun, D. Néron, P. Boucard, A model reduction technique based on the pgd for elastic-viscoplastic computational analysis, Computational Mechanics 51 (2012) 83-92. doi:https://doi.org/10.1007/ s00466-012-0706-x.

[34] P. Ladevèze, O. Loiseau, D. Dureisseix, A micro-macro and parallel computational strategy for highly heterogeneous structures, International Journal for Numerical Methods in Engineering 52 (12) (2001) 121-138. doi:10.1002/nme.274.

[35] P. Oumaziz, P. Gosselet, P.-A. Boucard, S. Guinard, A non-invasive implementation of a mixed domain decomposition method for frictional contact problems, Computational Mechanics 60 (5) (2017) 797-812. doi:10.1007/s00466-017-1444-x.

[36] P. Oumaziz, P. Gosselet, P. A. Boucard, M. Abbas, A parallel noninvasive multiscale strategy for a mixed domain decomposition method with frictional contact, Int. J. Numer. Methods Eng. 115 (8) (2018) 893-912. doi:10.1002/nme.5830.

[37] E. R. D, code-aster. URL www . code-aster .org

[38] D. Rixen, C. Farhat, A simple and efficient extension of a class of substructure based preconditioners to heterogeneous structural mechanics problems, Internat. J. Num. Meth. Engin. 44 (4) (1999) 489516. doi:10.1002/(SICI) 1097-0207(19990210)44:4<489::AID-NME514>3.0.C0;2-Z.

[39] A. Klawonn, O. B. Widlund, FETI and Neumann-Neumann iterative substructuring methods: Connections and new results, Commun. Pure Appl. Math. 54 (1) (2001) 57-90. doi:10.1002/ 1097-0312(200101) 54:1<57::AID-CPA3>3.0.CO;2-D.

[40] P. Gosselet, C. Rey, D. Rixen, On the initial estimate of onterface forces in FETI methods, Computer Methods in Applied Mechanics and Engineering 192 (2003) 2749-2764. doi:10.1016/ S0045-7825(03) 00288-3.

[41] K. Saavedra, O. Allix, P. Gosselet, J. Hinojosa, A.-E. Viard, An enhanced nonlinear multi-scale strategy for the simulation of buckling and delamination on 3D composite plates, Computer Methods in Applied Mechanics and Engineering 317 (2017) 952-969. doi:10.1016/j.cma.2017.01.015.

URL http://dx.doi.org/10.1016/j.cma.2017.01.015 